\title{
Temporal variability of Mrk 421 from XMM-Newton observations
}

\author{
W. Brinkmann ${ }^{1}$, I. E. Papadakis ${ }^{3,4}$, J. W. A den Herder $^{5}$, and F. Haberl ${ }^{2}$ \\ ${ }^{1}$ Centre for Interdisciplinary Plasma Science, Max-Planck-Institut für extraterrestrische Physik, Postfach 1312, \\ 85741 Garching, Germany \\ 2 Max-Planck-Institut für extraterrestrische Physik, Postfach 1312, 85741 Garching, Germany \\ 3 IESL, FORTH, 71110 Heraklion, Crete, Greece \\ 4 Physics Department, University of Crete, 71003 Heraklion, Crete, Greece \\ 5 SRON Laboratory for Space Research, Sorbonnelaan 2, 3584 CA Utrecht, The Netherlands
}

Received 12 November 2002 / Accepted 14 February 2003

\begin{abstract}
We present the results of a detailed spectral and temporal analysis of the currently available XMM-Newton observations of the bright BL Lac object Mrk 421 using mainly the EPIC-PN data. The source was found in various intensity states differing by up to a factor of five in count rates. In general, the source is more variable and shows a harder spectrum during higher intensities than when it is in lower states. The spectrum is very complex and cannot be fitted adequately by a broken power law or a continuously curved model. We find that the flux variations on time scales of $\gtrsim$ few thousand seconds are associated with significant and sometimes very complex spectral changes. The spectral variability rate is not the same in all cases and is correlated with the source flux state: the spectral variations per unit time increase with the source flux. The Cross-Correlation analysis shows that the soft and hard band light curves are often well correlated near zero lag, in other cases the hard band variations lead the soft band variations by typically $\sim 5 \mathrm{~min}$, in two cases we find the soft band leading the hard band variations. The delays appear to be correlated to the flares' duration: the shorter the flare, the smaller the delay.
\end{abstract}

Key words. BL Lacertae objects: individual: Mrk 421; X-rays: galaxies - radiation mechanisms: non-thermal

\section{Introduction}

BL Lacs are thought to be dominated by relativistic jets seen at small angles to the line of sight (Urry \& Padovani 1995), and their radio-through-X-ray spectra are well fitted by inhomogeneous jet models (Bregman et al. 1987). However, as BL Lac objects show remarkably featureless spectra in all energy bands the structure of the relativistic jets remains largely unknown and the measured spectra can be reproduced by models with widely different assumptions.

A substantial part of the information we possess about these objects is obtained from the analysis of the temporal variations of the emission and combined spectral and temporal information can greatly constrain the jet physics. Time scales are related to the crossing time of the emission regions which depend on wavelength and/or the time scales of physical processes like acceleration and radiative losses. The measured lags between the light curves at different energies as well as spectral changes during intensity variations allow to probe the physics of particle acceleration and radiation in the jet.

Recently, several extended observation campaigns on the prominent BL Lacs PKS 2155-304, Mrk 501, and Mrk 421 by ASCA and BeppoSAX, partly simultaneously with RXTE and $\mathrm{TeV}$ telescopes, have revealed that in general the X-ray

Send offprint requests to: W. Brinkmann, e-mail: wpb@mpe.mpg.de spectral index and the peak energy correlate well with the source intensity, although there are periods where in the same source this correlation is reversed (for a review see Pian 2002). The X-ray spectra are best described by curved and continuously steepening spectra with the Synchrotron peak energy typically at $\mathrm{keV}$ energies. The emission of the soft X-rays is generally well correlated with that of the hard X-rays and lags it by 3-4 ksec (Takahashi et al. 1996, 2000; Zhang et al. 1999; Malizia et al. 2000; Kataoka et al. 2000; Fossati et al. 2000a), however, significant lags of both signs were detected from several flares (Tanihata et al. 2001). Large flares with time scales of $\sim 1$ day were detected with temporal lags of less than 1.5 hours between X-ray and TeV energies (for Mrk 421 see Takahashi et al. 2000). For all three sources the structure function and the power density spectrum analysis indicates a rollover with a time scale of the order of 1 day or longer (Kataoka et al. 2001) which seems to be the time scale of the successive flare events. On shorter time scales only small power in the variability is found with a steep slope of the power density spectrum $\sim f^{-(2-3)}$ (Tanihata 2002).

These results were obtained from data with relatively low signal-to-noise ratio, integrated over wide time intervals (typically one satellite orbit) and by analyzing data from prominent flares with time scales of a day. Uninterrupted data with high temporal and spectral resolution can only be provided 
by XMM-Newton with its high sensitivity, spectral resolving power, and broad energy band. Correspondingly, from the first analysis of data taken with the XMM-Newton EPIC cameras from Mrk 421 Brinkmann et al. (2001) found an upper limit for a soft lag of $\tau \sim 265$ s only, and Edelson et al. (2001) give an upper limit to any lags of $|\tau| \lesssim 0.3 \mathrm{hr}$ from XMM-Newton observations of PKS 2155-304. They further suggest that previous claims of soft lags with time scales of $\sim$ hours might be an artifact of the periodic interruptions of the low-Earth orbits of the satellites every $\sim 1.6$ hours.

Mrk 421 is the brightest BL Lac object at X-ray and UV wavelengths and it is the first extragalactic source discovered at TeV energies (Punch et al. 1992). The nearby $(z=$ 0.031 ) object has been observed by essentially all previous $\mathrm{X}$-ray missions and shows remarkable X-ray variability correlated with strong activity at $\mathrm{TeV}$ energies (e.g., Takahashi et al. 1996; Maraschi et al. 1999). Early detailed studies with IUE and EXOSAT showed that the variability occurs on time scales of typically a day with an e-folding time scale of $\sim 5 \times 10^{4} \mathrm{~s}$ (George et al. 1988). The source shows a variety of X-ray states: a low, soft state $\left(f_{2-6 \mathrm{keV}} \lesssim 2 \times 10^{-11} \mathrm{erg} \mathrm{cm}^{-2} \mathrm{~s}^{-1}\right.$, $\Gamma \sim 2.8)$ where the source hardens when it brightens, a hard state $\left(f_{2-6 \mathrm{keV}} \gtrsim 8 \times 10^{-11} \mathrm{erg} \mathrm{cm}^{-2} \mathrm{~s}^{-1}\right)$ during which the spectral index remains at $\Gamma \sim 2$, and intermediate flux levels of $f_{2-6 \mathrm{keV}}=(3.6-5.2) \times 10^{-11} \mathrm{erg} \mathrm{cm}^{-2} \mathrm{~s}^{-1}$ found by ROSAT and Ginga (Makino et al. 1992; Tashiro 1994). The data indicated that the amplitude of the flux variations with time scales of a few hours get larger with increasing energy and the correlation between flux and spectral index was inconsistent with that observed earlier by EXOSAT.

Several multi-wavelength campaigns have been conducted to study possible time lags between the $\mathrm{X}$-ray band and TeV energies and to investigate the pronounced spectral evolution during flares seen in X-rays with ASCA and BeppoSAX (Macomb et al. 1995; Takahashi et al. 1996; Fossati et al. 1998; Maraschi et al. 1999). In general, the source shows a complex behavior. While Takahashi et al. (1996) found a lag of about $4000 \mathrm{~s}$ between the soft $(0.5-1.0 \mathrm{keV})$ photons and the hard band (2$7.5 \mathrm{keV}$ ), which was interpreted as an effect of radiative cooling, recent ASCA observations show both, positive and negative lags (Takahashi et al. 2000). BeppoSAX observations of a flare in April 1998, simultaneously observed at TeV energies, showed that the hard photons lag the soft ones by 2-3 ksec and that, while the light curve is symmetric at softest X-ray energies, it becomes increasingly asymmetric at higher energies with the decay being slower than the rise (Fossati et al. 2000a).

A broken power law model provides better fits to the ASCA data than a simple power law (Takahashi et al. 1996), but the $\chi_{\text {red }}^{2}$ is often unacceptable. With these models the break energy is at $\sim 1.5 \mathrm{keV}$, and the change of the power law index at the break point is $\Delta \Gamma \sim 0.5$.

With the wider energy range of BeppoSAX it became clear that these simple models are not adequate descriptions of the downward curved Synchrotron spectra (Fossati et al. 2000b) and continuously curved shapes had to be employed (Inoue \& Takahara 1996; Tavecchio et al. 1998). The Synchrotron peak energy varied between $0.4-1 \mathrm{keV}$, the spectral index at an energy of $5 \mathrm{keV}$ between $1.5 \leq \alpha \leq 2.2$. Both quantities are correlated with the X-ray flux: with increasing flux the synchrotron peak shifts to higher energies and the spectrum gets flatter at higher energies.

The analysis of the first XMM-Newton data demonstrated the high quality of the instruments (Brinkmann et al. 2001): for the first time the evolution of intensity variations could be resolved on time scales of $\sim 100 \mathrm{~s}$, temporal variations by a factor of three at highest X-ray energies were accompanied by complex spectral variations with only a small time lag of $\tau=265_{-102}^{+116} \mathrm{~s}$ between the hard and soft photons. In a recent paper Sembay et al. (2002) confirmed these short lags in an analysis of all previous XMM-Newton observations of Mrk 421. The authors show that the source exhibits a rather complex and irregular variability pattern - both, temporarily and spectrally.

In this paper we will present a more detailed analysis of the currently available XMM data, using the most recent response functions and re-processed data.

In particular, we will analyse the data on time intervals shorter than the individual observations as the source exhibits changes in its spectral properties on time scales down to a few thousand seconds.

We will first present the data, discuss the long-term temporal behavior of the object and demonstrate the spectral complexity of Mrk 421 in broad band spectral fits. We will then perform a temporal analysis of the hardness ratios for each pointing, adding in Sect. 4 a time resolved spectral analysis of two individual orbits. In Sect. 5 we present a Cross-Correlation analysis of the data in order to investigate the cross-links between the hard and soft band light curves. We then discuss the power density spectrum of Mrk 421 at high frequencies and briefly touch the non-linear properties of the light curves. Finally, a general discussion and conclusions will be given in Sect. 8 .

\section{The XMM-Newton observations}

Mrk 421 was observed during five orbits by XMM-Newton as a calibration source for the RGS for about $40 \mathrm{ksec}$ each. During most of these observations the PN camera was operated in Small Window (SW) mode, once in Timing mode (orbit 084), once in Large Window mode (orbit 440), usually with a thick filter. Only in orbit 259 a thin filter was used. The details of the observations are given in Table 1, where we first list the orbit in which the observation took place, the date of the observation, the instrument settings, the time over which the PN camera was "on" and then, in Col. 6, the life time of the detector. In Col. 7 we give a total exposure averaged count rate recorded from the chip as an indicator of the amount of data to be handled by the instrument electronics and as a rough measure of the source's intensity. No spatial nor quality selection for the photons were made for determining this quantity. The last column gives the average RGS count rate which is unaffected by pile up and the particular choice of the filter. For two observations no RGS data are available due to special modes of the instrument.

For the following analyses we will mainly use data from the PN camera. All data have been reprocessed using XMMSAS version 5.3.3 and the latest available versions of the response 
Table 1. Data of observations.

\begin{tabular}{llllcrrr}
\hline \hline Orbit & $\begin{array}{c}\text { Observing date } \\
\text { (UT) }\end{array}$ & PN mode & Filter & $\begin{array}{c}\text { On time } \\
\mathrm{s}\end{array}$ & $\begin{array}{c}\text { Live time } \\
(\mathrm{s})\end{array}$ & $\begin{array}{c}\text { count rate } \\
\text { on chip }\end{array}$ & $\begin{array}{r}\text { RGS } \\
\text { count rate }\end{array}$ \\
\hline 084 & May 25, 2000: 03:53-10:11 & Tim & Thick & 16279 & 16781 & 373.97 & $18.6 \pm 2.2$ \\
084 & May 25, 2000: 10:35-19:37 & SW & Thick & 30203 & 21159 & 300.13 & n/a \\
165 & Nov. 02, 2000: 00:10-10:25 & SW & Thick & 34560 & 24242 & 133.78 & $6.4 \pm 1.3$ \\
$171-1$ & Nov. 13, 2000: 22:22-11:20 & SW & Thick & 36553 & 25640 & 384.59 & $18.5 \pm 2.1$ \\
$171-2^{*}$ & Nov. 14, 2000: 13:54-01:51 & SW & Thick & 33384 & 23417 & 372.85 & $20.6 \pm 2.2$ \\
259 & May 08, 2001: 09:10-19:59 & SW & Thin & 36678 & 25727 & 407.42 & $14.0 \pm 1.9$ \\
$440-1^{*}$ & May 04, 2002: 16:49-03:03 & LW & Thick & 36715 & 34345 & 108.21 & $6.6 \pm 1.5$ \\
$440-2^{*}$ & May 05, 2002: 03:51-09:24 & LW & Thick & 17059 & 15954 & 78.01 & n/a \\
\hline
\end{tabular}

${ }^{\dagger}$ : Next day.

$*$ Source in off-set position.

matrices, released in April 2002 have been used. We selected photons with Pattern $\leq 4$ (i.e. singles and doubles) and quality flag $=0$. Although the frame time in the Small Window mode is only $\sim 5.7 \mathrm{msec}$ the source flux is so high that we find substantial pile up in the central pixels of the point spread function. We therefore disregarded a typically $2 \times 3$ RAW pixel region at the center of the source to minimize the effects of photon pile ups. In all analyses we used source photons extracted from a rectangular region of $30 \times 30 \mathrm{RAW}$-pixels, containing the source, apart from observation 171-2 when the source was observed off-center. The slightly widened point spread function required a larger extraction region as well as more pixels to be discarded in the peak of the PSF. During the first $\sim 23 \mathrm{ksec}$ of orbit 84 the PN camera was operated in timing mode for which we selected the photons from rows $28 \geq$ RAWX $\leq 48$ for our analysis. For the highest energies the background count rate sometimes reaches $\gtrsim 5 \%$ of the source counts; we therefore extracted hard backgrounds from the lower left (in RAW pixels) part of the chip with the same selection criteria as above. This region is still slightly contaminated by the extremely strong point source ( $\$ 2 \%$ of the source flux) but the corresponding count rates are considerably smaller than those obtained from this region during times of enhanced background activity.

\subsection{The light curves}

We calculated the $0.2-0.8 \mathrm{keV}$ and the $2.4-10 \mathrm{keV}$ light curves for all observations with the above photon selection criteria. Due to the high count rate from Mrk 421 the detector falls frequently into counting mode, which introduces gaps of typically $21 \mathrm{~s}$ duration in the light curves. Depending on the orbit, this can happen rather often; up to more than 480 times in observation 171-1. Other observations show additionally longer dead times of $\gtrsim 100 \mathrm{~s}$, and/or shorter interruptions of around one hundred frame times, so called FIFO overflow events. These data gaps result from the extremely high work load of the on-board electronics (FIFO overflows) and the limits of the telemetry rate (counting mode).

To minimize the effects of this non-uniform exposure we binned all light curves in $8 \mathrm{~s}$ bins and and disregarded periods of partial exposure. This binning still allows a statistically

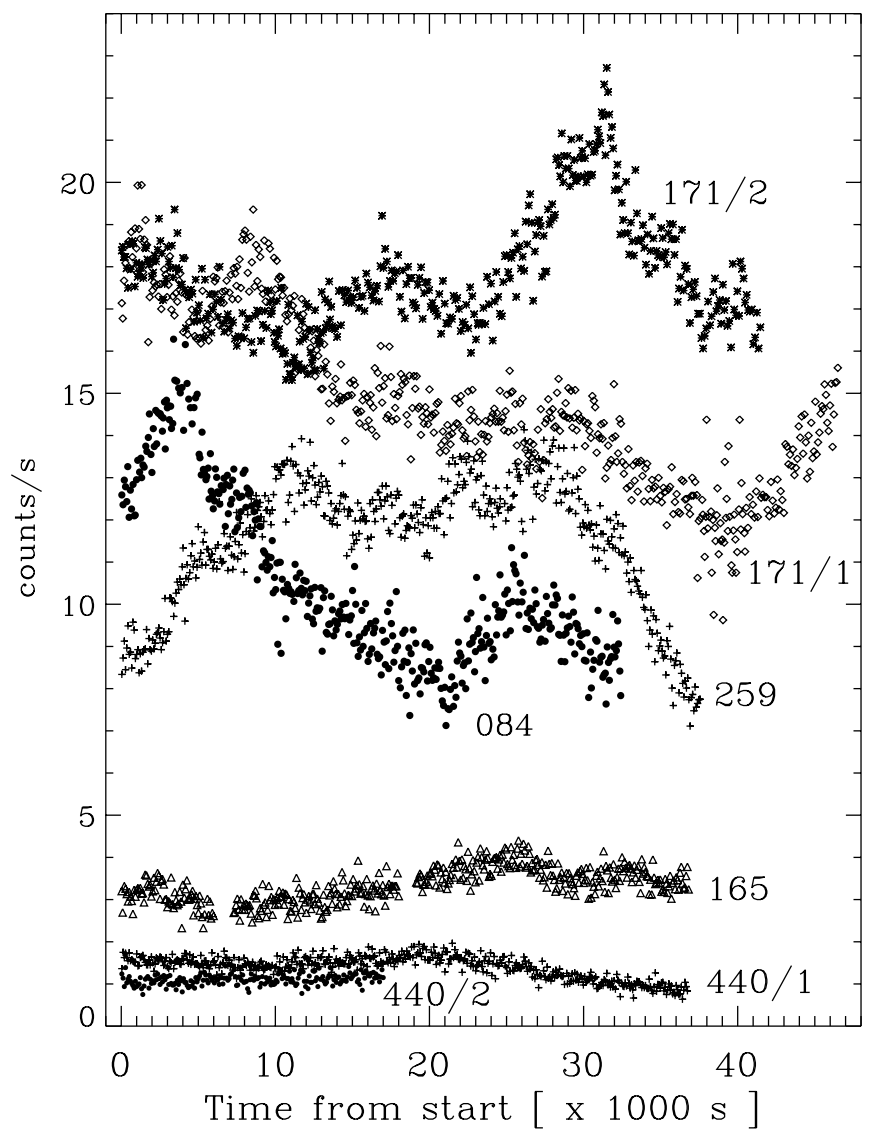

Fig. 1. Background subtracted, 2.4-10 keV PN light curves of Mrk 421. If no temporal gaps are encountered the time binning is $80 \mathrm{~s}$; times are counted from the beginning of the actual exposure. The curves are labeled by the orbit of the observation.

acceptable signal to noise ratio for both energy bands and provides a sufficiently large number of time bins for an advanced analysis.

In Fig. 1 we show the hard band light curves of all observations. Due to the completely different photon selection process for the timing mode observation it is not possible to determine the same fraction of photons from the point spread function as for the other observations and thus the absolute position (in 


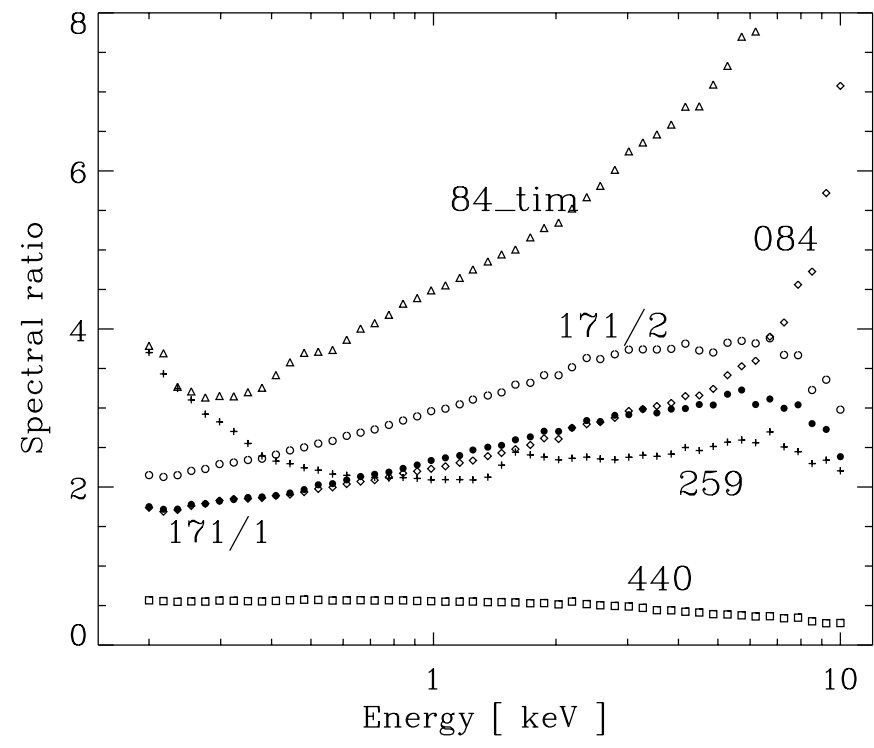

Fig. 2. Ratios of the raw spectra of individual observations divided by the spectrum of observation 165 .

count rate) in the plot is uncertain. In the following analysis this knowledge is not required as we use normalized light curves. The hard band was chosen to minimize the different transparencies of the filters and to allow a direct comparison of the intensity states of the source. There is still some smaller uncertainty in the count rates related to exact position of the point source in the disregarded central pixels. Please note that the "real" count rate is considerably higher (by $\gtrsim 45 \%$ ) as a large fraction of the source photons found in the center of the point spread function has been screened out.

For clarity we have binned the light curve in nominally $80 \mathrm{~s}$ bins. If data gaps were encountered the time bins are shorter which becomes apparent in the larger scatter of the data points. The light curves are characterized by long term trends lasting for time scales comparable to or longer than the length of the observations and, superimposed on this, of frequent flare like events of relatively regular shape with characteristic time scales of the order of a few thousand seconds. It should be noted that the light curve 171-2 follows, after a gap of $\sim 3$ hours for instrumental setup changes, directly after the end of 171-1. The same holds for the two observations in orbit 440, where the PN camera was operated in Large Window mode and the source was put at two different offset positions in the detector.

Figure 1 clearly shows that the source gets more variable on short time scales as the flux increases. Further, the spectrum hardens as demonstrated in Fig. 2 where we show the spectral ratios between the total raw count rates of the different observations, divided by the count rates of orbit 165 . The plot illustrates both, the spectral changes with respect to the spectrum of orbit 165 (discussed in more detail in the next section) as well as the changes in intensity. Note again, that the timing mode data from orbit 84 are selected in a different way so that the count rates appear to be higher; the spectral slope should not be affected substantially, apart from at low energies. The orbit 259 observation was performed with a thin filter which affects the low energy part of the spectrum $(\lesssim 1.5 \mathrm{keV})$ as can be seen from the ratios. Obviously, orbit 440 with the lowest count rate shows a spectrum softer than orbit 165 , all other spectra are harder. The differences consist of a generally harder/softer spectrum (in case of orbit 084 and 171/1 even nearly identical) up to energies of a few $\mathrm{keV}$ and dramatic orbit-to-orbit variations of the hard tail of the spectrum. These deviations start, basically, around 4-5 keV, i.e., where the spectrum breaks in the broken power law-fits. Finally, the spectra used are time averages over the whole observation; detailed spectral variations during the observations on time scales of $\gtrsim 1 \mathrm{ksec}$ are discussed in the next sections.

\subsection{Spectral analysis}

As mentioned above the spectral behavior of Mrk 421 is rather complex and with increasing data quality more sophisticated spectral models had to be used for the fits. Due to the extremely high photon statistics in the soft energy band the currently still existing calibration uncertainties in the PN detector response at low energies $(\$ 0.6 \mathrm{keV})$ inhibit reliable complex model fits over the total energy band (see Brinkmann et al. 2001 for a discussion).

This is demonstrated in fits to the data of orbit 165 which show very little variation in the hardness ratios during the observation (see Sect. 3). We selected only single events and excluded, as above, the center of the point spread function to avoid photon pile up. Figure 3 shows the fit to the total data set in the $0.2-12 \mathrm{keV}$ energy band with the continuously curved model (Fossati et al. 2000b). The fit is hardly acceptable $\left(\chi_{\text {red }}^{2}=1.25 / 1164\right.$ d.o.f. $)$ and show strong residuals at low energies. In a fit with a (currently not released) test version of an improved detector response matrix the residuals around $0.5 \mathrm{keV}$ are substantially reduced. However, the low energy slope and the break energy of the two fits differ considerably indicating that at present no reliable fit for data with such high signal to noise can be achieved at low energies. It must be noticed, however, that in absolute terms (ratio between model and data) the differences between model and data do not exceed $\sim 3 \%$.

The poor quality of the above fits is, however, not resulting from the relatively few energy channels at low energies but from the general mismatch of the model from the data. The systematic positive bump of the residuals at energies $\sim 3-6 \mathrm{keV}$ demonstrates that even the curved model is only an approximate representation of a more complex broad-band X-ray spectrum. A broken power law model does not provide an acceptable fit either $\left(\chi_{\text {red }}^{2}=1.24 / 1165\right.$ d.o.f. $)$; the break energy found is $E_{\mathrm{b}}=5.62 \pm 0.39 \mathrm{keV}$.

In Fig. 4 we show in the upper panel the broken power law fit to the data in the restricted $1.5-12 \mathrm{keV}$ energy band. The fit is excellent $\left(\chi_{\text {red }}^{2}=1.02\right.$ for 909 d.o.f.) with power law slopes of $\Gamma_{\text {soft }}=2.576 \pm 0.008, \Gamma_{\text {hard }}=2.873 \pm 0.049$, and a break energy of $E_{\text {break }}=4.94 \pm 0.28 \mathrm{keV}$. The absorption was fixed to the galactic value $\left(N_{\mathrm{H}}=1.66 \times 10^{20} \mathrm{~cm}^{-2}\right)$. In the middle panel we plot the ratio between the fitted model and the data, extrapolated to lowest energies. The deficit in the data of $20 \%$ at energies below $0.6 \mathrm{keV}$ clearly shows that the source spectrum 


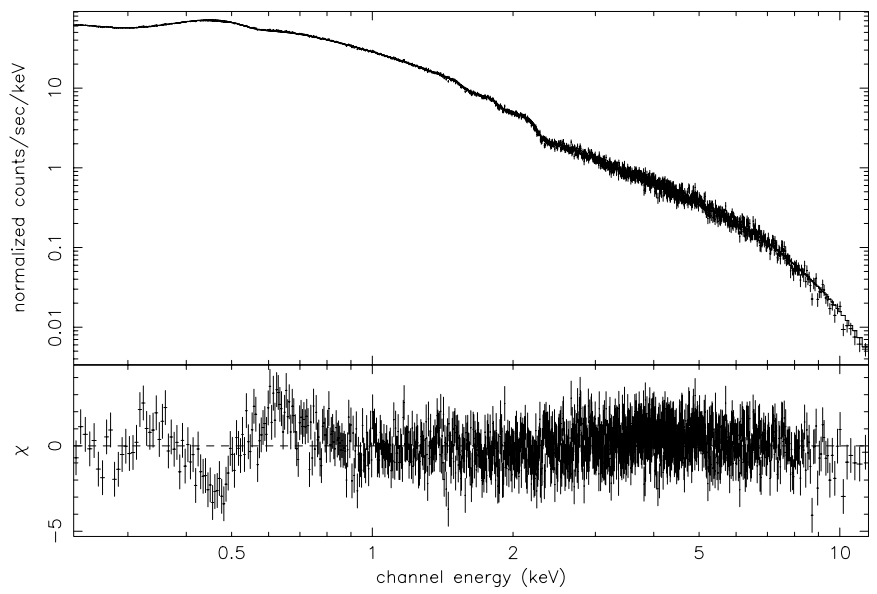

Fig. 3. Continuously curved model fit to the PN Small Window mode data of Mrk 421 during orbit 165 in the energy range $0.2-12 \mathrm{keV}$. The lower panel show the residual in units of $\delta \chi^{2}$ per energy bin.

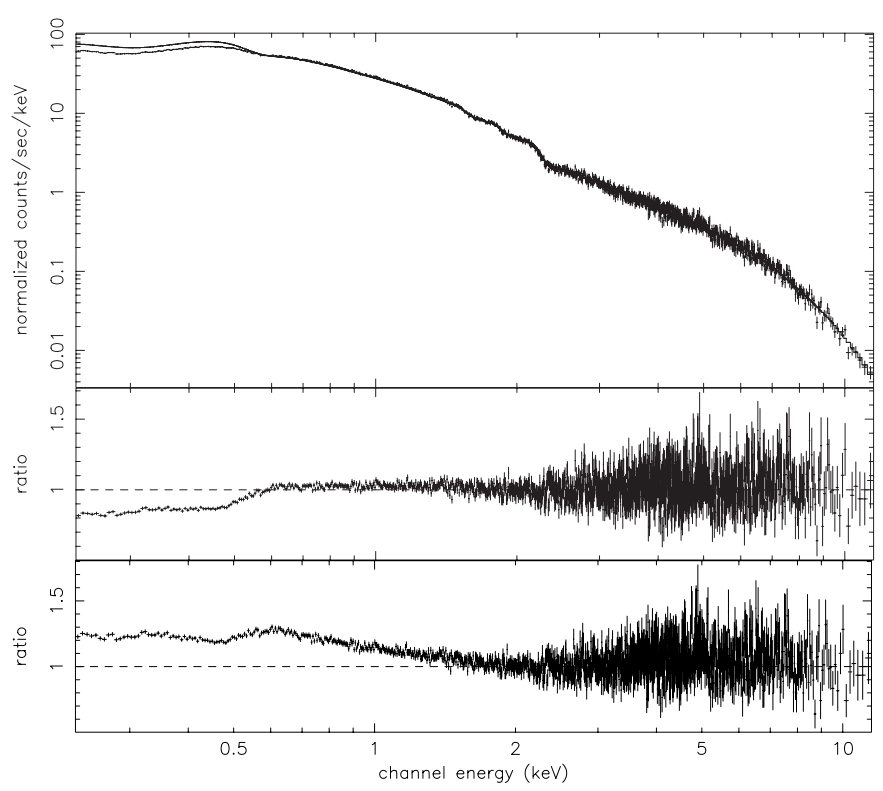

Fig. 4. Broken power law model fitted to the $1.5-12 \mathrm{keV}$ data during orbit 165 . The middle panel shows the ratio between model and data when the fitted model is extrapolated to lower energies. The lower panel shows the same ratio for the continuously curved best fit model.

must break again, below $1 \mathrm{keV}$. Correspondingly, using data at energies lower than $\sim 1.5 \mathrm{keV}$ decreases the quality of the fit considerably.

Fitting the same energy band $(1.5-12 \mathrm{keV})$ with the curved model results in an only slightly worse fit $\left(\chi_{\text {red }}^{2}=1.08\right.$ for 908 d.o.f.). The slopes are $\Gamma_{0.5 \mathrm{keV}}=2.20 \pm 0.06, \Gamma_{5.0 \mathrm{keV}}=$ $2.73 \pm 0.02$; however, the break energy $\left(E_{\mathrm{b}}=1.3 \mathrm{keV}\right)$ and the curvature index of 0.357 are only poorly constrained. The lower panel shows, again, the extrapolation of the ratio model/data to lower energies and finds a clear excess of the data over the model, i.e. at lower energies the spectral curvature is smaller than predicted by the model.

The high statistical significance of the PN data reveals that both spectral models, commonly used to fit the X-ray spectra of BL Lacs, are obviously insufficient to describe the
Table 2. Results for power law fits to the data of orbit 171-1 in limited energy bands, assuming galactic absorption $\left(N_{\mathrm{H}}=1.66 \times 10^{20} \mathrm{~cm}^{-2}\right)$.

\begin{tabular}{cccc}
\hline \hline $\begin{array}{c}\text { Energy band } \\
(\mathrm{keV})\end{array}$ & $\Gamma$ & $\begin{array}{c}\text { Norm } \\
(1)\end{array}$ & $\chi_{\text {red }}^{2} /$ d.o.f. \\
\hline $0.6-1.2$ & $2.25 \pm 0.01$ & $5.99 \pm 0.01$ & $0.997 / 120$ \\
$1.2-1.8$ & $2.32 \pm 0.02$ & $6.03 \pm 0.04$ & $1.21 / 121$ \\
$1.8-2.3$ & $2.40 \pm 0.05$ & $6.38 \pm 0.23$ & $1.32 / 97$ \\
$2.5-3.5$ & $2.21 \pm 0.04$ & $5.60 \pm 0.26$ & $0.98 / 199$ \\
$3.5-4.5$ & $2.39 \pm 0.07$ & $7.19 \pm 0.69$ & $1.03 / 218$ \\
$4.5-6.0$ & $2.47 \pm 0.08$ & $8.15 \pm 1.03$ & $1.04 / 298$ \\
$6.0-8.0$ & $2.59 \pm 0.11$ & $9.95 \pm 2.06$ & $0.99 / 213$ \\
$8.0-10.0$ & $2.77 \pm 0.28$ & $13.86 \pm 7.52$ & $0.99 / 70$ \\
$10.0-12.0$ & $2.82 \pm 0.78$ & $16.35 \pm 29.01$ & $1.24 / 17$ \\
\hline
\end{tabular}

(1): Normalization at $1 \mathrm{keV}$ in $10^{-2} \mathrm{ph} / \mathrm{keV} / \mathrm{cm}^{2} / \mathrm{s}$.

complex Mrk 421 spectra. As the count rates in the PN camera are rather high we were able to perform single power law fits over smaller energy bands to follow the changes of the spectral slope. We used the data of the first part of the bright orbit 171-1 (see Sect. 3.3). The results, given in Table 2 show a continuous steepening of the spectrum at higher energies, apart from the fit at energies around $\sim 2 \mathrm{keV}$ where we still find calibration uncertainties due to the edge structures of $\mathrm{Si}$ and $\mathrm{Au}$ in the residuals.

At lowest energies $(0.2-1.2 \mathrm{keV})$ we additionally tried broken power law fits with different instrument setups. In all three cases, the higher energy power law slope was nearly identical to the single power law slope (in Table 2) in the $0.6-1.2 \mathrm{keV}$ range. With the current $\mathrm{PN}$ response matrix the lower energy power law index was $\Gamma_{\text {low }}=1.74 \pm 0.02$ with a break energy of $E_{\text {break }}=0.65 \pm 0.01 \mathrm{keV}$; the (unpublished) test response matrix yielded $\Gamma_{\text {low }}=0.65 \pm 0.07$ and $E_{\text {break }}=0.48 \pm 0.01 \mathrm{keV}$. Both fits were unacceptable $\left(\chi_{\text {red }}^{2}>1.6\right)$. The broken power law fit to the RGS data in the $0.3-1.7 \mathrm{keV}$ range yielded $\Gamma_{\text {low }}=$ 1.88 and a break energy of $E_{\text {break }}=0.95 \mathrm{keV}$. Noticeable is that the pattern of the residuals of all three fits are systematically different as well.

These fits demonstrate that the currently used models are inadequate for a correct description of the curving spectrum; at least one more component seems to be required which must not necessarily be a power law. However, the presently existing uncertainties in the detector response inhibit the unambiguous determination of this component.

\section{Hardness ratios}

Considering the complex spectral behavior we will present in this section a more detailed analysis of the observed spectral variations using hardness ratios for the individual orbits. For every orbit we first present the soft and the hard, background subtracted, light curves binned in $80 \mathrm{~s}$ bins, and individually normalized to 1.0. Light curves normalized in this way have a variance equal to their rms amplitude, and provide a simple 
way to compare the amplitude of the variations in the soft and hard bands. In all plots we used the same scale for the normalized count rate and for the time axis $(\Delta T=50 \mathrm{ksec}$, as determined from the length of the longest observation). In this way, we can compare easily the variability behavior of the source (i.e. amplitudes, time scale lengths) during the different observations.

For each observation, we show the normalized soft and hard band light curves and the hardness ratio plots in the same Figures to investigate whether the spectral variations are associated with the respective flux variations. We generally find that when the source flux increases or decreases, the spectrum changes accordingly in a consistent way. Based on these "correlated" flux and spectral variations, we divide the observations into various sub-intervals of length $\sim 10-30 \mathrm{ksec}$. These divisions were made heuristically as the plots indicate that various source "states" can be rather easily identified during the individual observations. We use only large scale, large amplitude flux and spectral variations in the identification of individual "parts" in each observation, although in some cases, small amplitude, short-lived flux and spectral variations superimposed on the overall flux/spectral variability trends can also be observed. The sub-intervals identified in this section will be used later (Sect. 5) for the Cross-Correlation analysis between the soft and hard band flux variations, providing us a picture of the cross-links between the two bands which is totally different from the results of a Cross-Correlation of the whole light curves.

We computed the hardness ratios using the 80-s binned soft and hard band light curves, and the expression, $H R=$ [2.4-10 keV]/[0.2-0.8 keV]. By plotting $H R$ as a function of the total count rate $(0.2-10 \mathrm{keV})$, we are able to investigate whether the $H R$ variations are correlated with the source flux. For all plots we used the same scale for the $H R$ variations $(\triangle H R=0.2)$ and due to the small energy dependence of the XMM telescope over the point spread function, these hardness ratios of the various observations can be compared directly. One exception is orbit 259 , where the thin filter has been used, which is more transparent at low energies. Spectral simulations with the "typical" curved power law of Sect. 2.2 indicate a shift of -0.04 in the plotted value of the hardness ratio for the comparison with the thick filter observations. The other exception is the timing mode data of orbit 084 where noise contributes to the source flux at energies of $0.2-0.4 \mathrm{keV}$.

In Table 3 we list the mean count rate of total and the hard band (Cols. 2 and 3, respectively) for all orbits studied in this work. Columns 4 and 5 give the variability amplitudes $\mathcal{A}$ for the normalized soft and hard band light curves, respectively. This quantity is only a measure for the maximal variability of the corresponding orbit and is thus biased by the length of the exposure. Obviously, in none of the orbits the exposure is sufficiently long to cover all relevant variability time scales of the source. As the values listed in Table 3 show, the amplitude of the hard band variations is in all cases systematically larger than the soft band variability amplitude. The last two columns give the "spectral variability rate" $\mathcal{V}_{\mathrm{s}}$, i.e., the change of the hardness ratios per unit time for the rising and the decaying parts of the light curves (see Sect. 3.1).
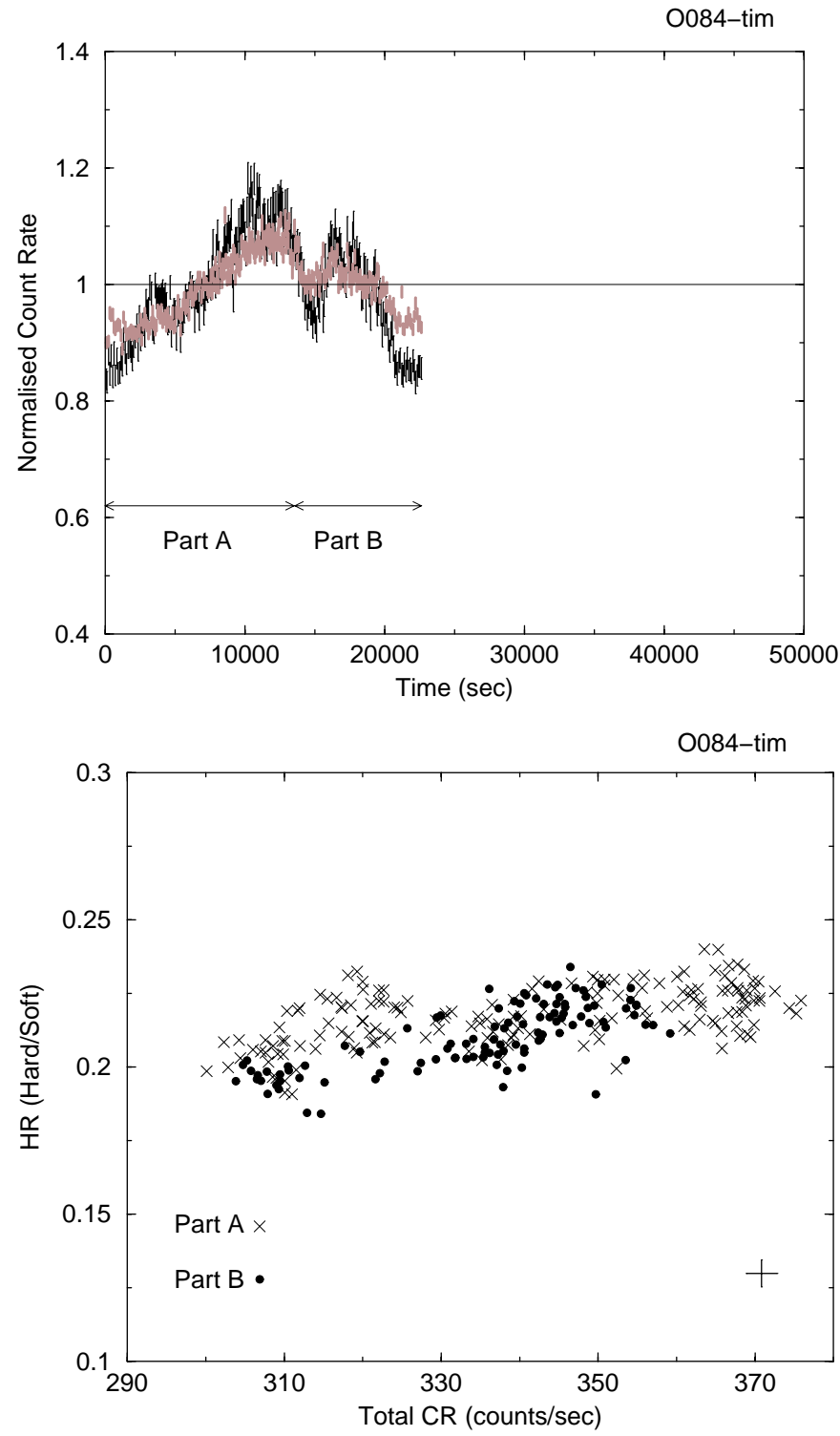

Fig. 5. In the upper and lower panels we plot the normalized hard (black) and soft (grey) light curves for orbit 084 (timing mode) and the hardness ratios $(H R)$ as a function of the total count rate, respectively. In the $H R$ plot, points plotted as crosses correspond to the first $\sim 13 \mathrm{ksec}$ part of the observation, when the total count rate was increasing. Points plotted as filled circles correspond to the remaining part of the observation, when the total band flux was decreasing. For clarity, in this and in the next 6 figures, we do not plot the errors of the points. Instead, we indicate in the right lower part of the $H R$ plot the average errors with solid crosses.

\subsection{Orbit 084}

In Fig. 5 we plot the normalized hard and soft band light curves of the first part of orbit 84 (timing mode) observation (black and grey lines, respectively). The variations observed in the hard band light curve appear in the soft band light curve as well. The main difference is their amplitude. We observe a $~ 30 \%$ and $20 \%$ max to min variation around the mean in the hard and soft band light curves, respectively. Because of this difference, we expect to detect flux related spectral variations. 
Table 3. Characteristics of the variability of the light curves.

\begin{tabular}{|c|c|c|c|c|c|c|}
\hline Orbit & $\begin{array}{l}C R_{\mathrm{tot}} \\
\mathrm{cts} / \mathrm{s}\end{array}$ & $\begin{array}{c}C R_{\mathrm{HB}} \\
\mathrm{cts} / \mathrm{s}\end{array}$ & $\begin{array}{c}\mathcal{A}_{\text {soft }} \\
\text { soft }\end{array}$ & $\begin{array}{c}\mathcal{A}_{\mathrm{h}} \\
\text { hard }\end{array}$ & $\begin{array}{c}\mathcal{V}_{\mathrm{s}} \\
\text { rising }\end{array}$ & $\begin{array}{c}\mathcal{V}_{\mathrm{s}} \\
\text { decaying }\end{array}$ \\
\hline \multirow[t]{2}{*}{ 84-tim } & 338.2 & 34.5 & 0.19 & 0.28 & $\begin{array}{c}0.08 \pm 0.01 \\
(\text { Part A) }\end{array}$ & - \\
\hline & & & & & - & $\begin{array}{c}0.18 \pm 0.02 \\
(\text { Part B })\end{array}$ \\
\hline \multirow[t]{2}{*}{84} & 71.1 & 10.5 & 0.25 & 0.68 & $\begin{array}{c}0.15 \pm 0.02 \\
(\text { Part A) }\end{array}$ & $\begin{array}{c}0.21 \pm 0.01 \\
\quad(\text { Part A) }\end{array}$ \\
\hline & & & & & $\begin{array}{c}0.27 \pm 0.04 \\
(\text { Part B })\end{array}$ & $\begin{array}{c}0.16 \pm 0.03 \\
(\text { Part B })\end{array}$ \\
\hline \multirow[t]{3}{*}{165} & 52.9 & 3.3 & 0.27 & 0.42 & $\begin{array}{c}0.06 \pm 0.02 \\
(\text { Part A) }\end{array}$ & $\begin{array}{c}0.06 \pm 0.02 \\
(\text { Part A) }\end{array}$ \\
\hline & & & & & $0.06 \pm 0.02$ & - \\
\hline & & & & & (Part B) & - \\
\hline \multirow[t]{2}{*}{$171-1$} & 162.2 & 14.8 & 0.13 & 0.49 & - & $\begin{array}{c}0.32 \pm 0.02 \\
\quad(\text { Part A) }\end{array}$ \\
\hline & & & & & $\begin{array}{c}0.15 \pm 0.02 \\
\quad(\text { Part C })\end{array}$ & $\begin{array}{c}0.15 \pm 0.02 \\
\quad(\text { Part C })\end{array}$ \\
\hline $171-2$ & 157.2 & 13.7 & 0.15 & 0.51 & $\begin{array}{c}0.44 \pm 0.05 \\
\quad(\text { Part B })\end{array}$ & $\begin{array}{c}0.52 \pm 0.05 \\
\quad(\text { Part B })\end{array}$ \\
\hline \multirow[t]{2}{*}{259} & 132.6 & 8.8 & 0.19 & 0.52 & $\begin{array}{c}0.15 \pm 0.01 \\
(\text { Part A) }\end{array}$ & - \\
\hline & & & & & - & $\begin{array}{c}0.18 \pm 0.01 \\
(\text { Part B })\end{array}$ \\
\hline $440-1$ & 27.5 & 1.4 & 0.37 & 0.68 & - & $0.05 \pm 0.01$ \\
\hline $440-2$ & 16.7 & 0.7 & - & 0.26 & - & - \\
\hline
\end{tabular}

In the lower panel of Fig. 5 we plot the $H R$ values as a function of the count rate in the total $0.2-10 \mathrm{keV}$ band. We have identified two parts in the light curves plotted in the upper panel of Fig. 5 (Part A and B, shown with the underlying arrows) when the source flux increased and then decreased. The $H R$ values which correspond to Part A and B are plotted with crosses and filled circles, respectively. We observe significant variations which are correlated with the source flux in both cases. During Part A, as the source flux increases, the spectrum becomes "harder/flatter" (HR increases), while during Part B, as the flux decreases, the spectrum becomes "softer/steeper" ( $H R$ decreases). Both, the hardening and softening of the spectrum appear to follow similar paths in the $H R$ diagram. To investigate this issue further, we normalized the total band count rate values to their mean, and fitted straight lines to the $H R$ variations in Part A and B. The slope of such a line, the spectral variability rate $\mathcal{V}_{\mathrm{s}}$, is listed in Cols. 6 and 7 of Table 3 . It is proportional to the rate of the spectral variations, i.e., the slope indicates the amplitude of the $H R$ variations per "unit" flux variation. The best fitting slopes are $0.08 \pm 0.01$ and $0.18 \pm 0.02$ for Part A and B, respectively, indicating that the spectrum softens at a faster rate than it hardens.

In Fig. 6 we plot the same quantities for the second part of the orbit 84 observation. The observational gap between the end of the timing mode operation (Fig. 5) and the start of the current Small Window mode data is $\sim 1515 \mathrm{~s}$. The light curves look similar but the amplitudes of the variations are different. We observe a $\sim 70 \%$ and $\sim 30 \%$ max to min variation around
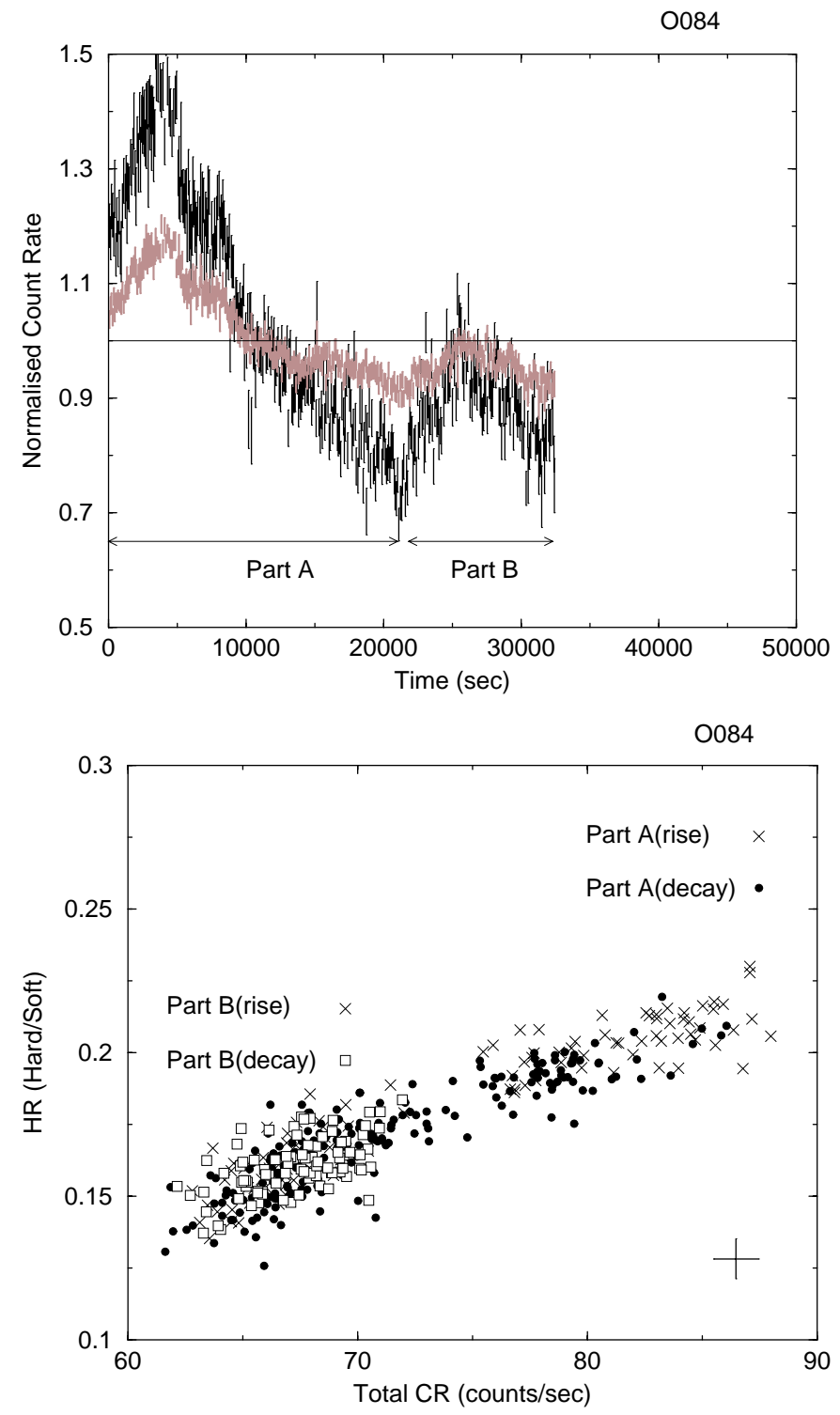

Fig. 6. As Fig. 5 but for the Small Window mode data of orbit 084. In the $H R$ plot, the crosses correspond to the first $\sim 4 \mathrm{ksec}$ of the observation, when the source flux was increasing. Filled circles correspond to the following $\sim 18 \mathrm{ksec}$ part of the observation when the total band count rate was decreasing. The crosses in the left part of the $H R$ plot correspond to the $\sim 4 \mathrm{ksec}$ long part of the observation ( $\sim 22 \mathrm{ksec}$ after the start of the observation) when the source flux increased again, while open squares show the $H R$ variations during the last part of the observation when the source flux decreased once more.

the light curve mean in the hard and soft band light curves, respectively.

As a result, significant, large amplitude $H R$ variations are also observed (lower panel in the same figure). Comparing the average $H R$ values between the orbit $84(\sim 0.17)$ and the orbit 84 timing mode observations $(\sim 0.21)$ we conclude that the source during the orbit 84 observation moves into a "softer" state than during the timing mode observation. Points shown with crosses in the right part of the $H R$ diagram correspond to the $H R$ variations during the rising part of a flare like event, detected in the first $\sim 4 \mathrm{ksec}$ part of the observation. The spectrum 

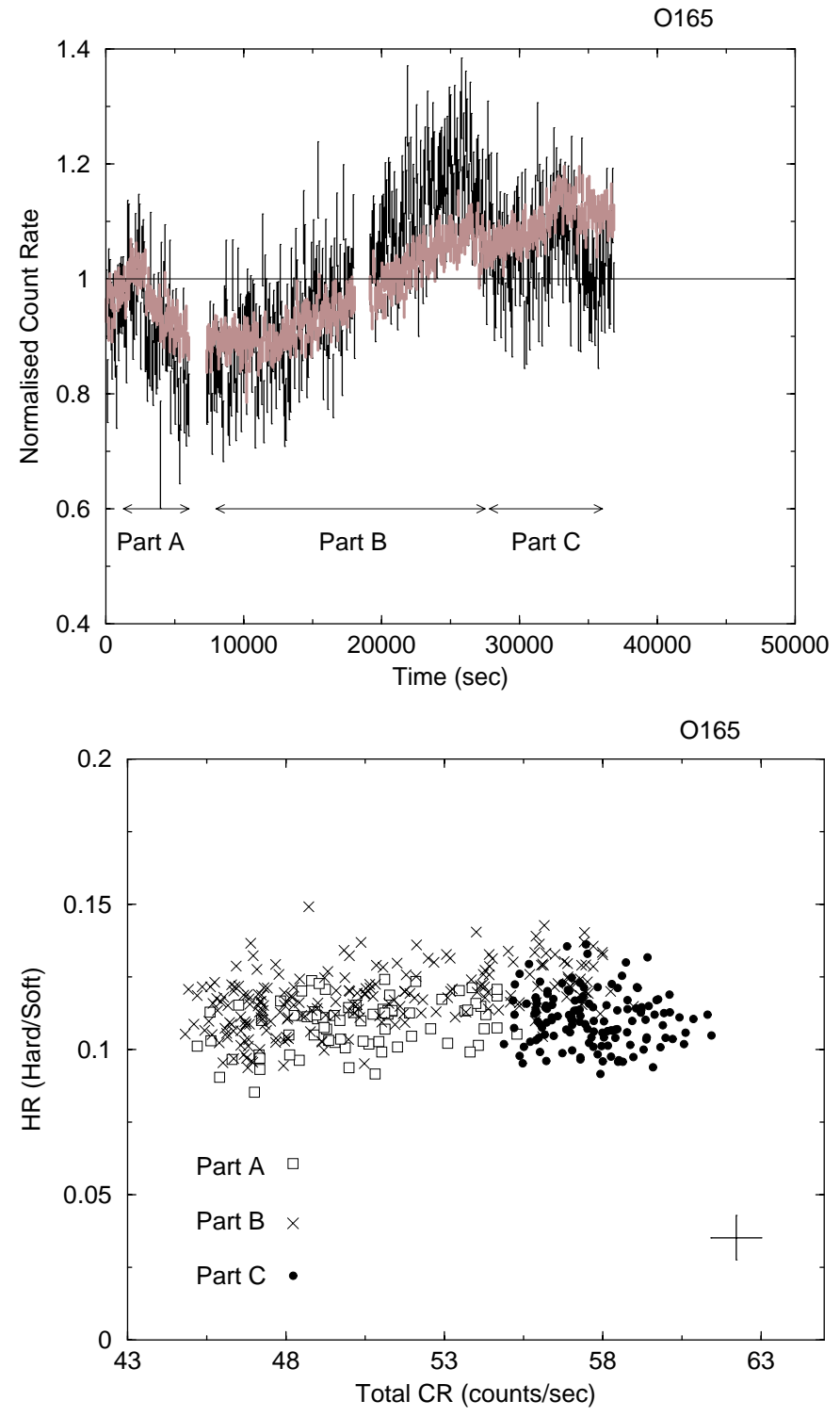

Fig. 7. The same as Fig. 5 but for orbit 165 . The open squares in the $H R$ plot correspond to the first $\sim 5 \mathrm{ksec}$ part of the observation, where a flare appears in both the hard and soft bands (as clearly visible in the upper panel). Crosses show the $H R$ variations for the following $\sim 20 \mathrm{ksec}$, during which the total band flux increased. Finally, the filled circles correspond to the last $\sim 10 \mathrm{ksec}$ part of the observation, during which the total count rate decreased slightly.

flattens significantly as the flux increases. During the following $\sim 15 \mathrm{ksec}$, the source flux decreases and the spectrum steepens accordingly. Towards the end of the observation another flare is detected ("Part B"). The $\mathcal{V}_{\mathrm{s}}$ values for the rising and the decaying parts of the light curves are listed in Table 3. Again, the spectrum hardens and then softens as the flare evolves. During Part A, the rate of the spectral changes is slightly larger during the softening of the spectrum, while in Part B the spectrum evolves faster during the rising than the decaying part of the flare.

\subsection{Orbit 165}

Figure 7 shows the normalized hard and soft band light curves during the orbit 165 observation when Mrk 421 was in a very low intensity state. As in the previous observations, similar variations are observed in the two light curves, but their amplitudes are different (the min to max amplitude around the mean is $\sim 30 \%$ and $\sim 25 \%$ for the hard and soft band light curves, respectively).

The average $H R$ value is $\sim 0.11$, significantly smaller than that of the two orbit 84 observations. This implies that the source is in a significantly softer state during this observation. The amplitude of the $H R$ variations are also significantly reduced, compared to orbit 84. During the first part of the observation ("Part A"), a flare is detected in both bands. The $H R$ increases and then decreases during the rising and decaying part of the flare (points shown with open squares in the lower panel of Fig. 7). During "Part B" the total flux increases and the spectrum hardens. The $\mathcal{V}_{\mathrm{s}}$ values listed in Table 3 suggest a similar rate of spectral variations during both, the softening and the hardening of the spectrum. Finally, during the last part of the present observation the source flux was variable (albeit with a small amplitude), but not associated with $H R$ changes as well.

\subsection{Orbit 171-1}

The soft and hard band variations show large differences in their amplitudes during the orbit 171-1 observation (upper panel in Fig. 8). The hard light curve exhibits a $\sim 50 \% \mathrm{~min}$ to max variation around the mean, compared to a $\sim 15 \%$ variation observed in the soft band. Apart from the large difference in the variability amplitude, the light curves are quite similar. During the first part of the observation ("Part A" in Fig. 8) two flares are detected in the hard light curve and a similar pattern with a much smaller amplitude appears in the soft band as well. In the following part of the observation ("Part B") the light curves show a rather complex behavior. On average, the soft flux appears to increase while the hard flux decreases slightly. Similar, smaller amplitude variations superimposed on these general trends appear in both light curves. Finally, a decay and a subsequent rise of the flux appears in both bands in the last part of the observation ("Part C"), although the amplitude of this feature is significantly larger in the hard band light curve.

The large differences between the variability amplitudes in the soft and hard band light curves result in large $H R$ variations as well (lower panel in Fig. 8). In fact, these $H R$ variations show the largest amplitude of all the observations presented in this work. During Part A, the total band flux decreases, and the spectrum softens. This is consistent with the trend observed in other observations (i.e. steepening of the spectrum as the source flux decreases). However, the softening of the spectrum happens at a rate which is faster than the rate of many similar events in other observations as the $\mathcal{V}_{\mathrm{s}}$ value, listed in Table 3 , suggests. During the decaying part of Part C, the source flux decreases and the spectrum steepens again, at a rate which is slower than the spectral softening rate during Part A. During the rising phase of Part $\mathrm{C}$, the spectrum flattens accordingly. 
O171-1

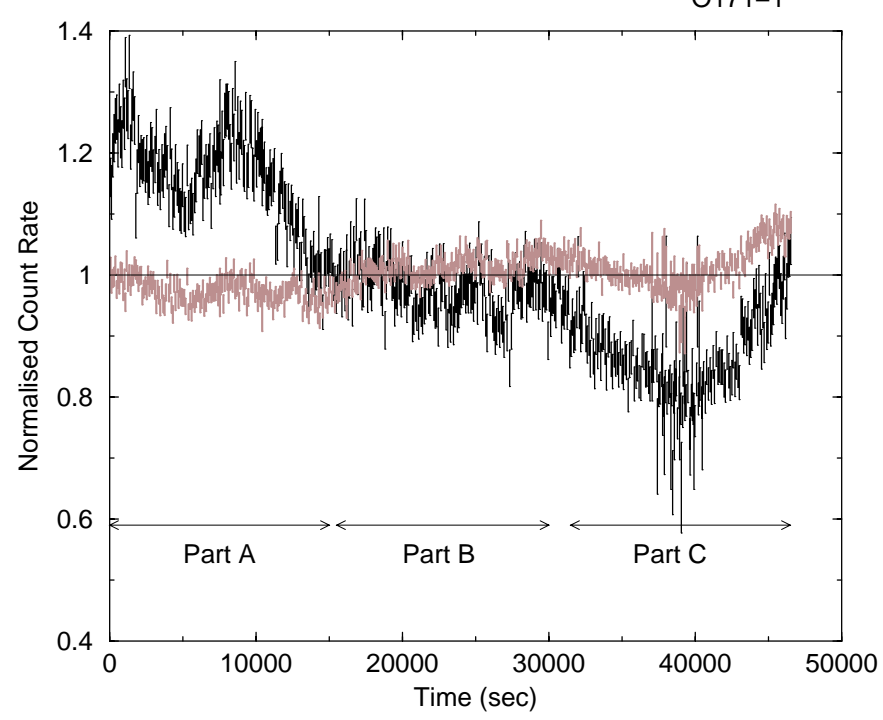

0171-1

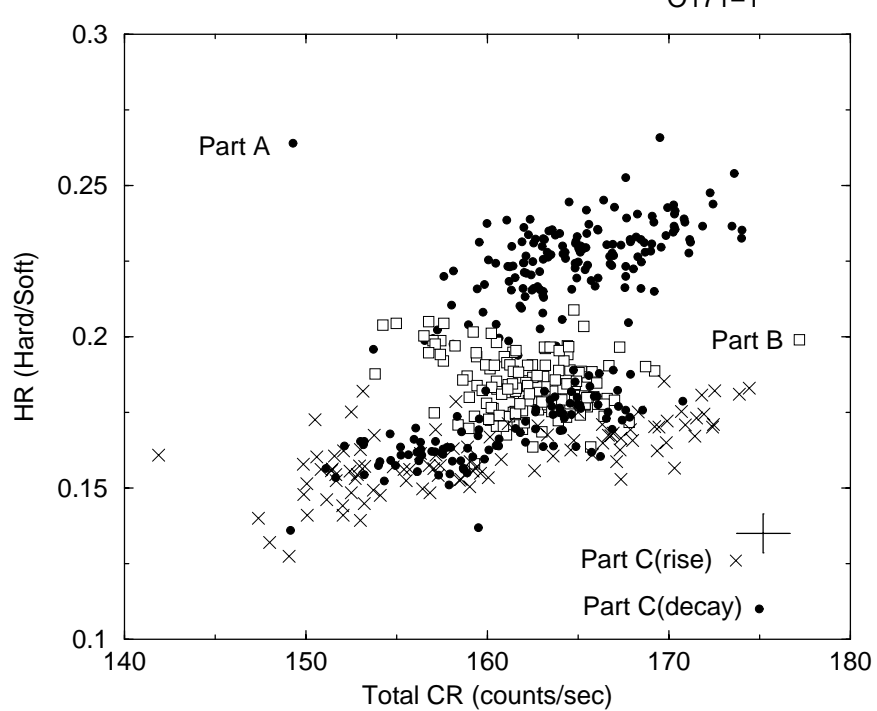

Fig. 8. The normalized hard and soft band light curves (upper panel) and $H R$ plot for orbit 171-1 (lower panel). This observation shows the strongest $H R$ variations. Filled circles in the upper right part of the $H R$ plot correspond to the first $\sim 15 \mathrm{ksec}$ part of the observation ("Part A" in the upper panel) when the total band count rate was decreasing. During "Part B", the total count rate increased, and the corresponding $H R$ variations are shown by open squares. Filled circles and crosses in the lower left part of the $H R$ plot correspond to the the first and second part part of the last $\sim 17 \mathrm{ksec}$ of the observation, when the source flux decreased and then increased again.

The best fitting slope of $0.15 \pm 0.02$ is listed in Table 3. Finally, during Part B, although the source flux increases, the $H R$ decreases, i.e. the spectrum steepens (open squares in the lower panel of Fig. 8). This is opposite to what is found in any other case where we observe the total source flux increasing.

\subsection{Orbit 171-2}

At the end of the orbit 171-1 observation a slight re-orientation of the instrumental setups led to an observing gap such that
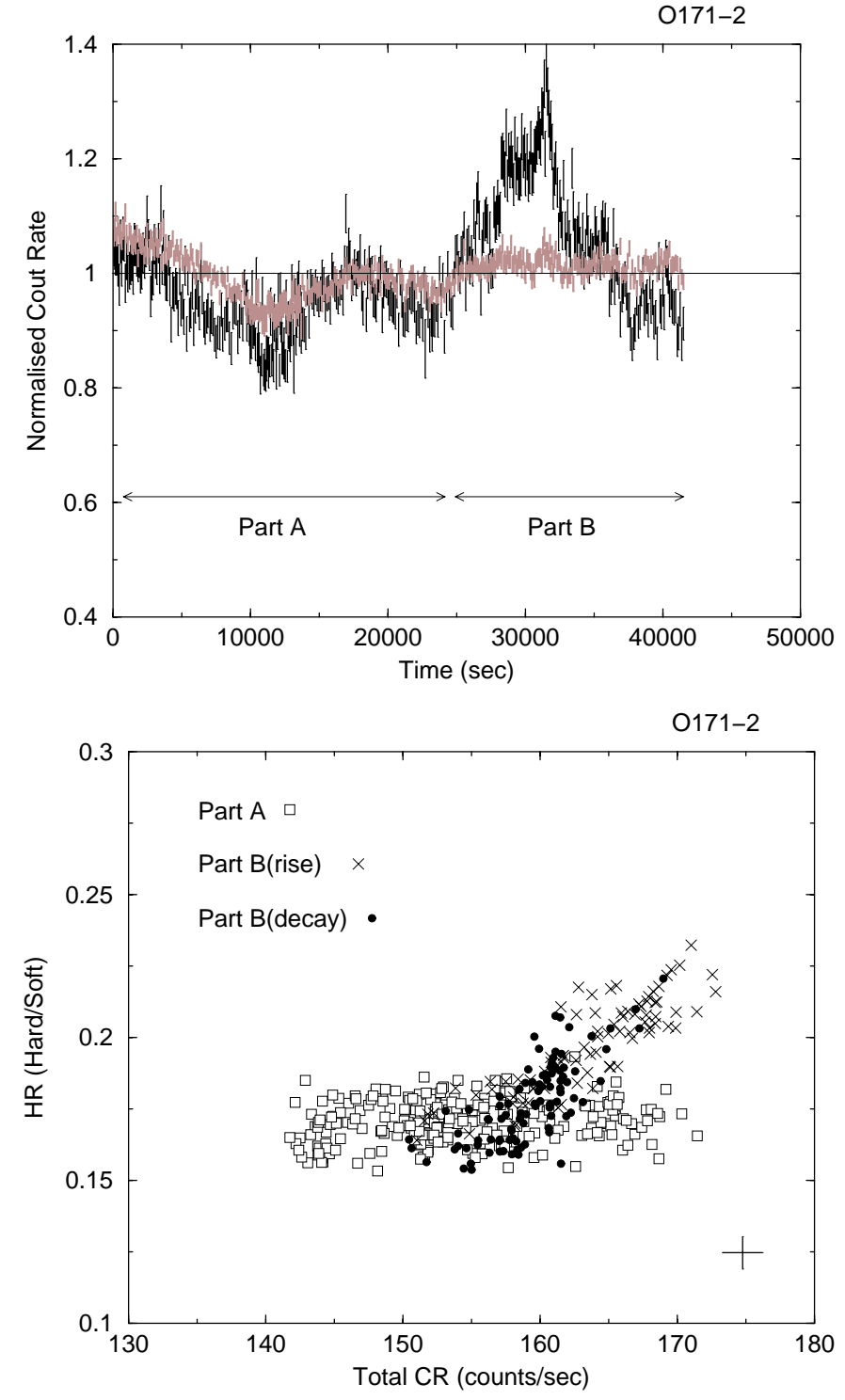

Fig. 9. The normalized hard and soft band light curves and $H R$ plot for the orbit 171-2 observation (upper and lower panels, respectively). Open squares in the $H R$ plot correspond to the $H R$ variations during the first part of the observation (indicated as "Part A" in the upper panel). Crosses and filled circles show the $H R$ variations during the rising and decaying part, respectively, of the prominent hard band flare which appears in the last part of the observation.

the 171-2 observation started $\sim 10666 \mathrm{~s}$ later. The soft and hard band light curves of the orbit 171-2 observation show differences not only in their variability amplitude, but also in the presence of significant hard band variations which are difficult to detect in the soft band light curve. The two normalized light curves are shown in the upper panel of Fig. 9. During the first part of the observation ("Part A") the light curves appear to be quite similar. The max to min variation around the mean is $\sim 20 \%$ and $\sim 15 \%$ in the hard and soft band light curves, respectively and the corresponding $H R$ values (green points in the $H R$ plot) show only small amplitude variations. However, during the last part of the observation ("Part B"), a major flare appears in the hard band. This flare is almost entirely absent in 
the soft band light curve. The soft band flux does not remain constant, however, it is not clear if the small amplitude variations are associated with the hard band flare. This flare is accompanied by strong $H R$ variations (crosses and filled circles in the $H R$ plot). Although the amplitude of the spectral variations is not larger than the variations observed during the orbit 171-1 observation, the rate of the spectral evolution is significantly higher than in any other of the observations analyzed in this paper (see the respective $\mathcal{V}_{\mathrm{s}}$ values in Table 3 ). The decaying part shows the largest $\mathcal{V}_{\mathrm{s}}$ value (i.e., the fastest evolution rate), which agrees with the fact that the flare decays faster than it rises (Fig. 9).

\subsection{Orbit 259}

The normalized hard and soft band light curves from the orbit 259 observation are shown in Fig. 10 (upper panel). At the beginning of the observation the source flux increased, then it stayed roughly constant and decreased during the last part of the observation. Both light curves exhibit similar variability patterns, of different amplitude. We observe a $~ 60 \%$ hard band max to min variation around the mean which is significantly larger than the respective soft band variation $(\sim 20 \%)$.

The $H R$ variations show flux dependent trends which are similar to those observed in the previous observations. During the first part of the observation ("Part A"), when the source flux increases, the spectrum hardens (crosses in the HR plot). At the end of the observation ("Part B") the spectrum steepens as the flux decreases. As the $\mathcal{V}_{\mathrm{s}}$ values suggest (Table 3 ), the rate of the spectral variations is slightly higher during the flux decrease. In between the rising and decaying parts of the flare, the source flux remained roughly constant and no significant spectral variations are detected (points plotted with open squares in Fig. 10).

Please note that this observation was performed with a thin filter; all others with a thick filter. Due to the larger transparency of the thin filter at lower energies the hardness ratios in Fig. 10 have to be shifted by $\sim 0.04$ to higher values (in case of a curved spectrum as determined in Sect. 2.2 for the data of orbit 165) for a comparison with the hardness ratios from the other observations.

\subsection{Orbit $440-1$}

During orbit 440-1 Mrk 421 was in the second lowest flux state of all XMM-Newton observations, and in a very "soft" spectral state as well. The average $H R$ value is $\sim 0.08$ (see lower panel in Fig. 11), the second smallest value after orbit 440-2 (see below). As a result of the low count rate, the light curves are quite "noisy" (especially in the hard band) as Fig. 11 shows. During the first part of the observation ("Part A" in the figure), both the soft and hard band light curves show only small amplitude variations, while at $\sim 20 \mathrm{ksec}$ after the beginning of the observation, the flux decreases by $\sim 55 \%$ and $\sim 35 \%$ in the hard and soft band light curves, respectively. The rate of decrease in the hard band light curve is faster than the respective decay rate in the soft band. As a result, we expect the spectrum to become softer
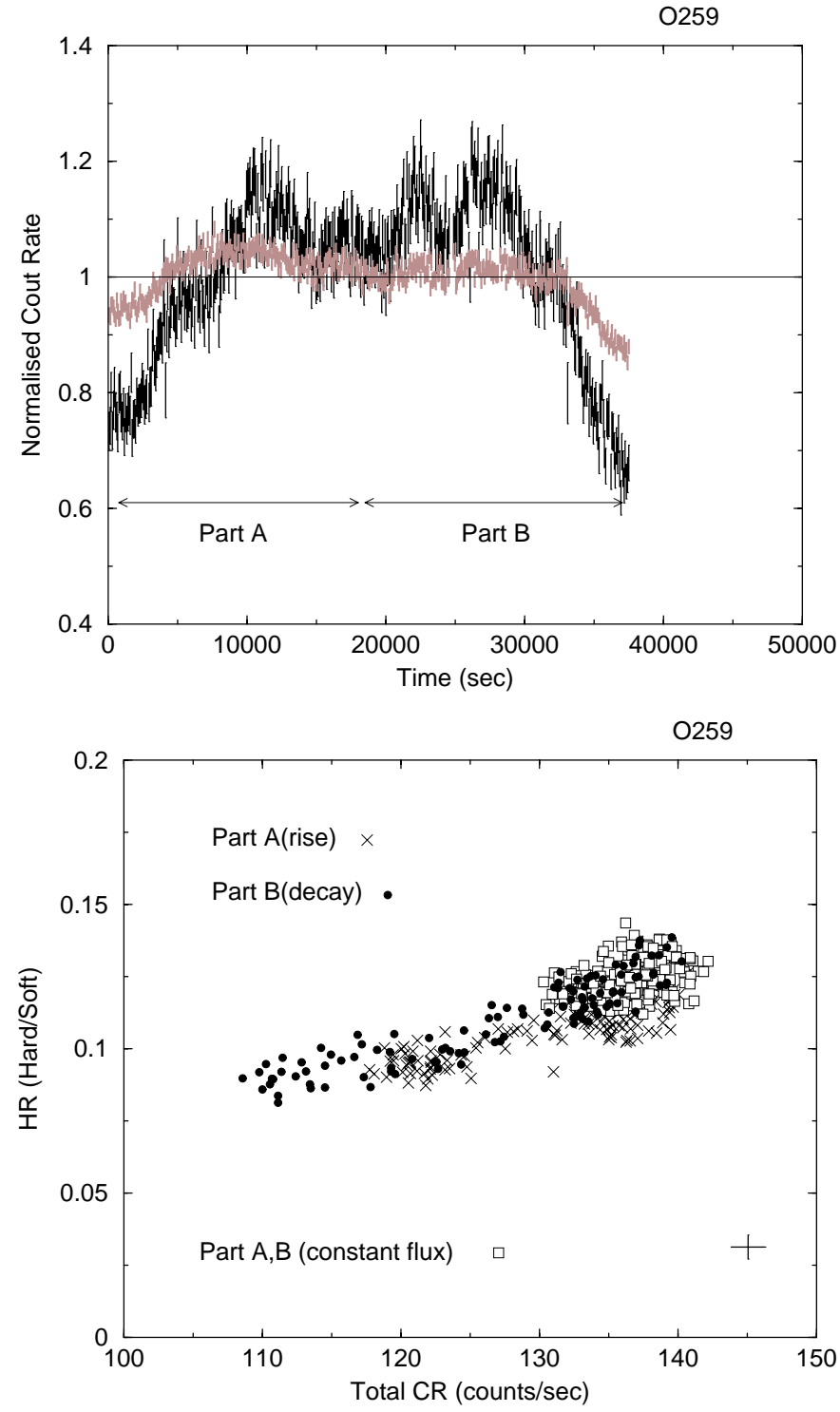

Fig. 10. The same as the previous figures, for orbit 259. Crosses and filled circles in the $H R$ plot correspond to the rising and decaying flux parts in the beginning and towards the end of the observation, respectively. Open squares show the $H R$ variations during the middle part of the observation, when the total flux remained roughly stable.

as the source flux decreases. This is confirmed by the $H R$ plot, shown in the lower panel of Fig. 11. Filled circles the plot correspond to the spectral variations of the second part of the observation ("Part B" in the upper panel), and show clearly that, as the flux decreases, $H R$ decreases (i.e. the spectrum steepens) accordingly. The respective $\mathcal{V}_{\mathrm{s}}$ value (Table 3 ) is one of the lowest slopes observed. Open squares in the $H R$ plot imply that the low amplitude variations during the first part of the observation occur without significant changes of the spectral shape.

\subsection{Orbit $440-2$}

Mrk 421 was in its lowest flux state among the XMM-Newton observations during orbit 440-2 (which started 5300 s after 

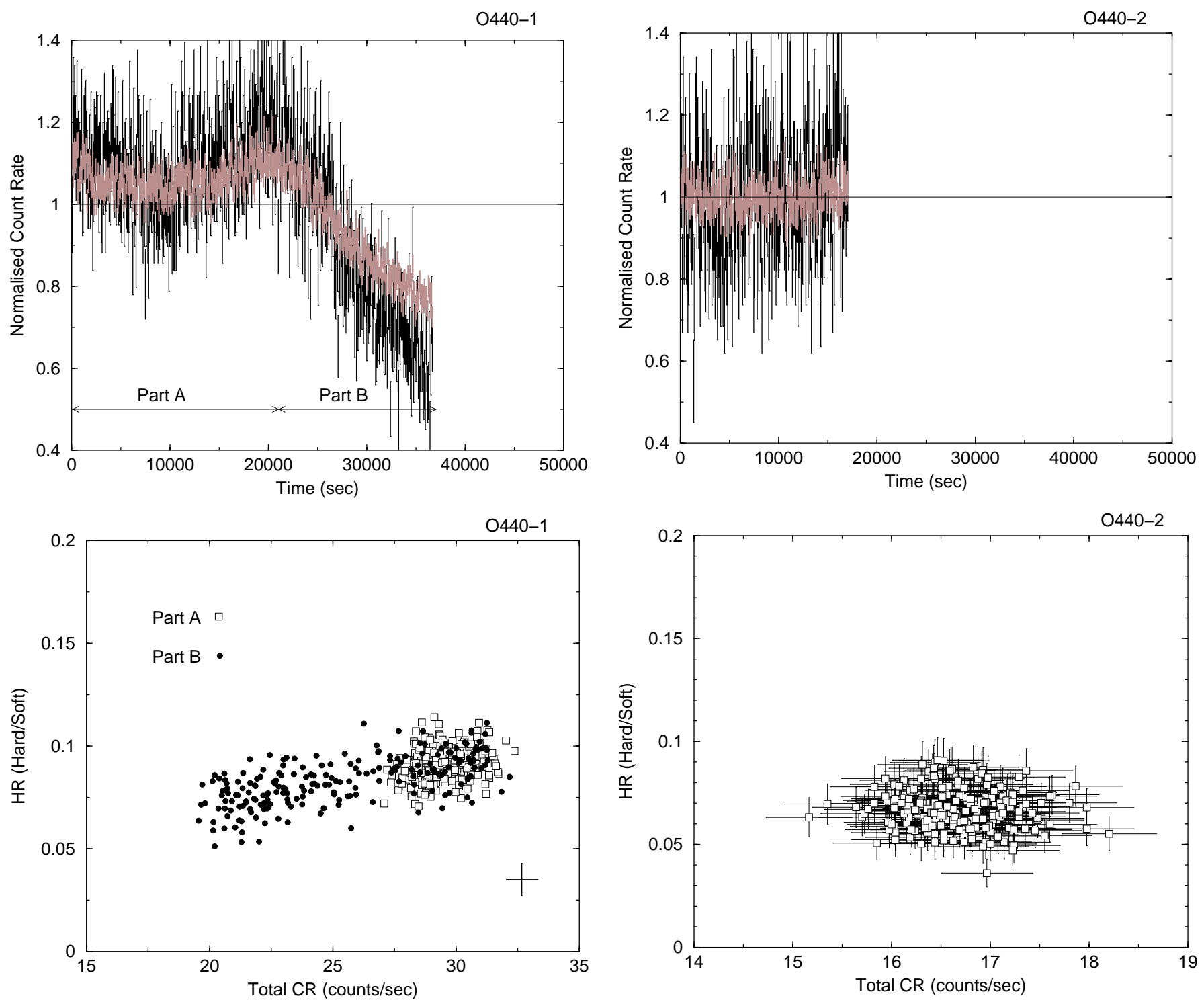

Fig. 11. Normalized light curves and $H R$ plot for the orbit 440-1 observation (upper and lower panels, respectively). The open squares in the $H R$ plot show that during the beginning of the observation ("Part A" in upper panel) the source does not show spectral variations. Filled circles correspond to the last part of the observation ("Part B") when the source flux was decreasing.

orbit 440-1). The source was at its softest state as well. The average $H R$ value is $\sim 0.07$ (see the lower panel in Fig. 12), which is the smallest value of all XMM-Newton observations presented in this work. The normalized hard and soft band light curves plotted in the upper panel of Fig. 12 do not show any significant variations (as verified by the $\chi^{2}$ fitting of a constant to the data). As a result, we do not observe spectral variations either, the $H R$ plot (lower panel in Fig. 12) clearly shows the absence of significant spectral variability during this observation.

\section{Spectral variability}

A hardness ratio analysis provides for short time scales a sensitive method to study spectral variations, but as only broadband properties are used it does not reveal directly the detailed

Fig. 12. Normalized light curves and $H R$ plot for the orbit 440-2 observation (upper and lower panels, respectively). The source is at a very low intensity state, and the light curves in both bands (upper panel) do not show any statistically significant variations. As a result, no spectral variability is observed either, as seen in the $H R$ plot.

spectral changes responsible for the observed changes of the hardness ratios. We therefore looked in more detail into the two extreme observations, the orbits 165 and 171-1. We fitted each of the sub-intervals indicated in Figs. 7 and 8 with a broken power law model, assuming Galactic absorption, in the energy range $1.5-12 \mathrm{keV}$. For the period $171-1 / \mathrm{C}$ we selected the data stretch covering $10 \mathrm{ks}$ around the minimum. The results of the fits are given in Table 4.

In all cases the broken power law provides an acceptable fit; the data set of orbit 165/A seem to suffer a bit from the lowest photon statistics. The break energy stays relatively constant around $\gtrsim 4.7 \mathrm{keV}$, the high energy slope is always steep, around $\Gamma \sim 2.8$. The low intensity orbit 165 clearly shows a steeper low energy power law with less variance than orbit 171, where the slope is flattest in the highest state. The main cause of intensity variations thus seems to be a hardening of the low energy 
Table 4. Results for broken power law fits for Mrk 421 in the energy band 1.5-12.0 keV, assuming Galactic absorption.

\begin{tabular}{lcccc}
\hline \hline $\begin{array}{c}\text { Observation } \\
\text { period }\end{array}$ & $\Gamma_{\text {low }}$ & $\begin{array}{c}E_{\text {break }} \\
\text { keV }\end{array}$ & $\Gamma_{\text {high }}$ & $\chi_{\text {red }}^{2} /$ d.o.f. \\
\hline $\mathrm{O} 165 / \mathrm{A}$ & $2.62 \pm 0.01$ & $6.25 \pm 0.51$ & $3.19 \pm 0.22$ & $1.029 / 475$ \\
$\mathrm{O} 165 / \mathrm{B}$ & $2.53 \pm 0.01$ & $4.69 \pm 0.29$ & $2.79 \pm 0.05$ & $0.949 / 873$ \\
$\mathrm{O} 165 / \mathrm{C}$ & $2.60 \pm 0.01$ & $4.92 \pm 0.46$ & $2.82 \pm 0.07$ & $0.940 / 673$ \\
\hline $\mathrm{O} 171-1 / \mathrm{A}$ & $2.22 \pm 0.01$ & $4.66 \pm 0.11$ & $2.64 \pm 0.03$ & $1.071 / 1204$ \\
$\mathrm{O} 171-1 / \mathrm{B}$ & $2.38 \pm 0.01$ & $4.77 \pm 0.11$ & $2.83 \pm 0.03$ & $1.031 / 1154$ \\
$\mathrm{O} 171-1 / \mathrm{C}$ & $2.47 \pm 0.01$ & $4.27 \pm 0.13$ & $2.88 \pm 0.03$ & $1.015 / 969$ \\
\hline
\end{tabular}

power law when the source increases its intensity; the shape of the harder part of the spectrum remains relatively stable. As the flux between orbits changes much more than can be caused by the hardening of the soft part of the spectrum the normalization of the spectrum has to change as well. A detailed and reliable analysis of the very low energy part of the spectrum could possibly clarify the situation, but this has to await further improvements of the detector responses.

\section{Cross-correlation analysis}

In order to investigate the cross-links between the hard and soft band light curves we estimated their Cross-Correlation Function $(C C F)$, using in this case light curves with 8-s long bin size. We chose to work with light curves which have a small bin size for two reasons. Firstly, because of the occurrences of non-uniform exposure in the XMM light curves if a larger bin size is used (see Sect. 2.1). Secondly, a Cross-Correlation analysis between soft and hard band light curves for four of the eight observations that we study in this work has already been performed (Sembay et al. 2002). The results showed that, if there exist inter-band delays, they are very small. For that reason, a light curve with a small bin size is necessary to sample the fast variations in both bands and determine accurately possible delays that may exist between them.

We calculated the sample Cross-Correlation Function, $C C F(k)$, as follows:

$$
C C F(k)=\frac{\sum_{t}\left(x_{\mathrm{soft}}(t)-\bar{x}_{\mathrm{soft}}\right)\left(x_{\mathrm{hard}}(t+k)-\bar{x}_{\mathrm{hard}}\right)}{N(k)\left(\sigma_{\mathrm{soft}}^{2} \sigma_{\text {hard }}^{2}\right)^{1 / 2}},
$$

$k=0, \pm \Delta t, \ldots, \pm(N-1) \Delta t$

The summation goes from $t=\Delta t$ to $(N-k) \Delta t$ for $k \geq 0$ and from $t=(1-k) \Delta t$ to $N \Delta t$ for $k<0(\Delta t=8 \mathrm{~s}$, and $N$ is the total number of points in the light curve). Due to the missing points, the summation was done over those pairs of time points, $(t, t+|k|)$, for which both the corresponding observations have been recorded and the sum was divided by the number of pairs included, i.e. $N(k)$. The variances in the above equation are the source variances, i.e. after correction for the experimental variance. Significant correlation at positive lags means that the soft band variations are leading the hard band.

First, we computed the $C C F$ (up to lags \pm 4 hrs) using the whole length (i.e. total) soft and hard band light curves.
Table 5. Cross-correlation analysis results. $C C F_{\max }$ is the maximum $C C F$ value, and $k_{\max }$ the lag at which this maximum appears (i.e. the "delay"). Both results were estimated using a model fitting procedure (see text for details).

\begin{tabular}{lccc}
\hline \hline Observation & $C C F_{\max }$ & $\begin{array}{c}k_{\max } \\
(\mathrm{s})\end{array}$ & Probability \\
\hline Orb84 $_{\mathrm{tm}} /$ PartA & 0.80 & $-433_{-162}^{+164}$ & $P_{\mathrm{kmax}<0}=99.7 \%$ \\
Orb84 $_{\mathrm{tm}} /$ PartB & 0.69 & $+103_{-94}^{+104}$ & - \\
Orb84/PartA & 0.90 & $-234_{-129}^{+125}$ & $P_{\mathrm{kmax}<0}=96.8 \%$ \\
Orb84/PartB & 0.52 & $+68_{-119}^{+120}$ & - \\
Orb165/PartA & 0.66 & $-98_{-191}^{+166}$ & - \\
Orb165/PartB & 0.91 & $-675_{-228}^{+251}$ & $P_{\mathrm{kmax}<0}=99.5 \%$ \\
Orb171-1/PartA & 1.00 & $-11_{-203}^{+178}$ & - \\
Orb171-1/PartC & 0.89 & $263_{-139}^{+149}$ & $P_{\mathrm{kmax}>0}=98.1 \%$ \\
Orb171-2/PartA & 0.90 & $-331_{-199}^{+200}$ & $P_{\mathrm{kmax}<0}=96.2 \%$ \\
Orb259/PartA & 0.90 & $336_{-147}^{+141}$ & $P_{\mathrm{kmax}>0}=99.1 \%$ \\
Orb259/PartB & 0.96 & $-156_{-156}^{+88}$ & - \\
Orb440-1/PartB & 0.92 & $-9_{-126}^{+130}$ & - \\
\hline
\end{tabular}

The resulting $C C F$ s are shown in the upper panels of Figs. 13 to 19 , for the observations during the corresponding orbits discussed above. The Cross-Correlation analysis of the orbit 84 , 165, 171 and 259 observations has already been presented by Sembay et al. (2002). Despite the differences of the energy bands chosen, and the use of newly processed data in this work, our results are in good agreement with their "soft/hard" CCF s (shown in their Fig. 3). We find that the soft and hard band light curves are well correlated during the orbit 84 and 259 observations. The maximum $C C F$ values $\left(C C F_{\max }\right)$ appear near zero lag. Slight asymmetries towards negative or positive lags (orbit 84 and 259, respectively) are also observed. The orbit $165 C C F$ is very broad, with no well defined peak and a strong asymmetry towards negative lags is observed. The orbit 171-1 CCF is unusual. An anti-correlation is observed at all lags between $\pm 4 \mathrm{hrs}$, with a broad peak at lags $\sim-1-1.5 \mathrm{hrs}$.

The orbit 84 (timing mode), 171-2, and 440-1 CCFs are presented in this work for the first time. The former shows a strong peak $\left(C C F_{\max } \sim 0.8\right)$ at zero lag and an asymmetry towards negative lags. The $C C F$ peak is smaller in the case of the orbit 171-2 observation $\left(C C F_{\max } \sim 0.6-0.7\right)$. This is expected because of the apparent absence in the soft band light curve of the strong flare which appears in the hard band towards the end of this observation (see Fig. 9). The $C C F$ of orbit 440-1 appears symmetric around zero lag, showing a strong peak of $C C F_{\max } \sim 0.95$. For the orbit 440-2 observation, we could not compute the $C C F$ due to the lack of significant flux variations. However, a different picture for the soft and hard band cross-links is revealed when we compute the $C C F$ s using only sub-parts of the total light curves.

In the second and third panel (from top) of Fig. 13 we present the $C C F$ of the two parts of the light curve shown in Fig. 5. The $C C F$ s are computed up to $\pm 1 \mathrm{hr}$ lags (this is the 

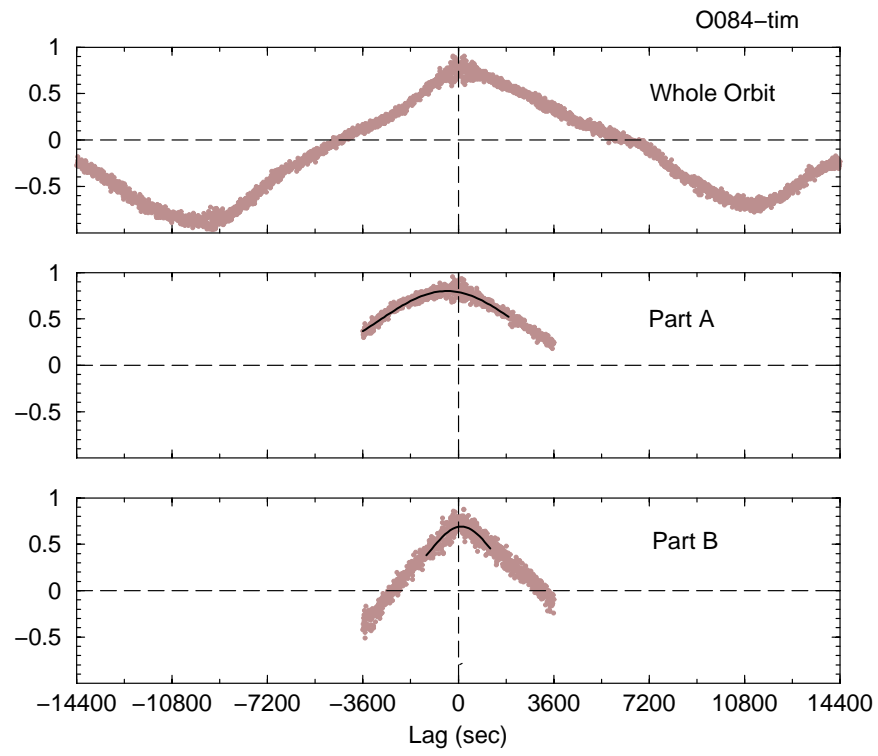

Fig. 13. $C C F$ plots for the orbit 84 (timing mode) observation. In the upper panel we plot the $C C F$ estimated using the $8 \mathrm{~s}$ binned soft and hard band light curves. In the lower two panels we plot the $C C F$ estimated using Part A and B light curves, indicated with the underlying arrows in Fig. 5. In all plots, positive lags correspond to the soft band leading the hard band variations. The solid lines in the lower panels indicate the best fitting skew Gaussian model curves to the $C C F$ s (see text for details).

case for the $C C F$ of the various parts of all the light curves presented in this section). During Part A the source flux increases, and the spectrum flattens, while during Part B the flux decreases and the spectrum steepens. The $C C F$ peak in both cases is similar $\left(C C F_{\max } \sim 0.7 / 08\right)$, and appears near zero lag. We fitted the $C C F$ s near their peak with a function consisting of an asymmetric (skewed) Gaussian which has the same peak but different standard deviations. The best fitting models are also shown in the figure (solid black lines). The best fitting results $\left(C C F_{\max }\right.$, and $k_{\max }$, i.e. the lag at which $C C F_{\max }$ is located) are listed in Cols. 2 and 3 of Table 5. The errors on $k_{\max }$ were estimated using the Monte Carlo methods of Peterson et al. (1998); they represent the $68 \%$ confidence limits on the best fitting values. We also list in the fourth column the probability that $k_{\max }$ is different from zero (either positive or negative). We consider as significant probabilities which are higher than $95 \%$ and we only list the results in these cases.

The results given in Table 5 imply that the soft and hard band variations are well correlated in both parts of the orbit 84 (timing mode) observation. There are no significant delays during the second flare detected in this observation (Part B). However, there is a significant probability that during the first flare-like event (Part A) the soft band variations are delayed with respect to the hard band variations by $\sim 7 \pm 2.7 \mathrm{~min}$.

In the second and third panel (from top) of Fig. 14 we plot the $C C F$ of the two parts of the orbit 84 (SW mode) light curves shown in Fig. 6. The first Part Coincides with the large amplitude flare at the beginning of the observation which appears in both bands and is associated with a flattening/steepening of the spectrum as the source flux increases/decreases. The
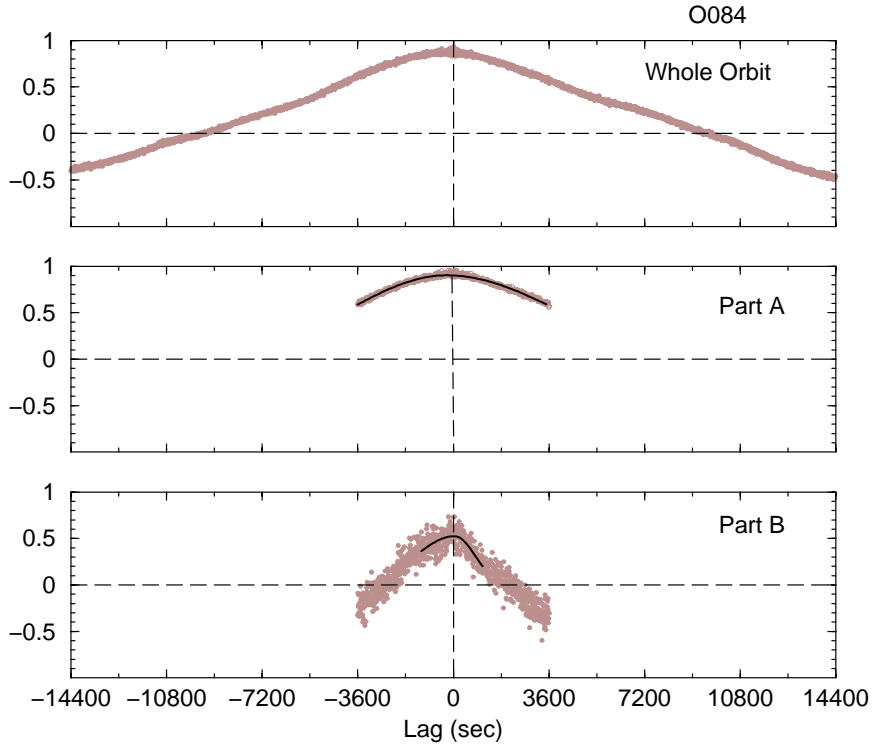

Fig. 14. Same as Fig. 13 for the data of orbit 84 (Small Window mode).
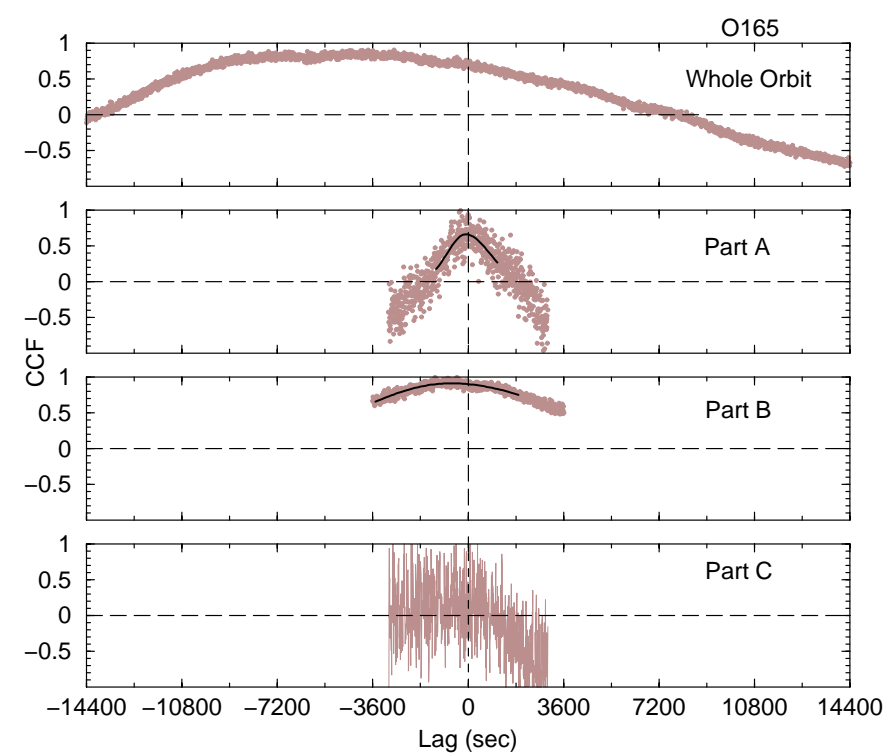

Fig. 15. Same as Fig. 13 for the data of orbit 165 .

second part includes the second flare which is detected at the end of the observation and which shows similar spectral variations. The soft/hard band correlation is very good during Part A $\left(C C F_{\max } \sim 0.9\right)$. We also find significant evidence that the hard band leads the soft band variations by $\sim 4 \pm 2 \mathrm{~min}$. The Part B light curves are less well correlated, and no significant delays are detected.

In Fig. 15 we plot the $C C F$ s of the three parts in the orbit 165 light curves (see Fig. 7). These $C C F$ s are significantly different from the $C C F$ of the total light curves, indicating that the "real" nature of the cross-links between the two bands can not be investigated properly only by computing the $C C F$ of the total light curves. During Part A a small amplitude flare appears in both bands. No spectral variations are observed, and the $C C F$ shows that the two light curves are reasonably well correlated $\left(C C F_{\max } \sim 0.7\right)$. The $C C F$ is noisy, probably 


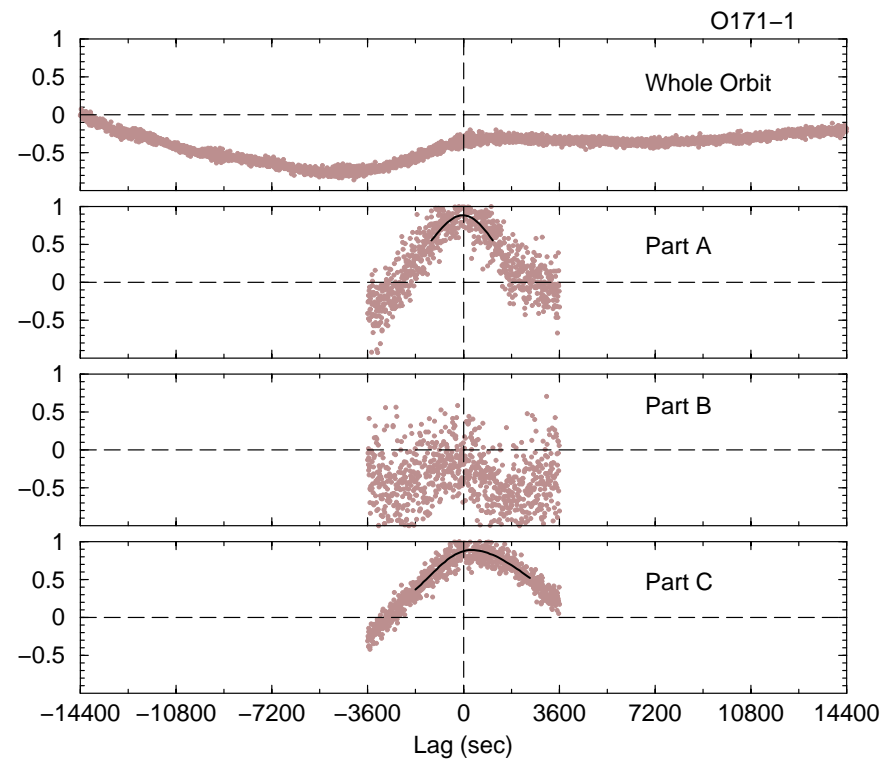

Fig. 16. The $C C F$ plots for the orbit 171-1 light curves. As in the previous figures, the lower panels show the $C C F$ of various parts of the observation.

because of the small number of data points in Part A. No significant lags are detected. During Part B the source flux increases, the spectrum becomes harder at a slow rate, and the two light curves are well correlated, as Fig. 7 shows; this impression is confirmed by the $C C F$ analysis $\left(C C F_{\max } \sim 0.9\right)$. A significant delay of the soft band variations by $\sim 11 \pm 4 \mathrm{~min}$ is detected. Finally, the $C C F$ of Part $C$ is very noisy. This is partly due to the small number of points in Part $\mathrm{C}$, and to the differences in the light curves, as can been seen in Fig. 7. Although similar small amplitude variations are observed in both bands, the soft band light curve shows a trend of increasing flux while the hard band light curve appears to be on an approximately constant flux level.

In Fig. 16 we plot the $C C F$ of the three parts in the Orb 171-1 light curves shown in Fig. 8. As in the orbit 165 observation, the $C C F$ s of the individual parts are significantly different from the $C C F$ of the total light curves. The anticorrelation shown in the $C C F$ of the total light curves is caused by the fact that, overall, the soft band flux increases throughout the observation while the hard band flux decreases. However, when we compute the $C C F$ for the three sub-parts, a different picture emerges. During Part A the source flux decreases, while during Part $\mathrm{C}$ the source flux first decreases and then increases again. The spectral variations follow the "normal" pattern (spectral hardening/softening when the flux increases/decreases, respectively; see Sect. 3). The $C C F$ s show that the soft and hard band light curves during these periods are well correlated, opposite to what is observed when the total light curves are used. The Part A CCF is noisy, but a positive peak at lag zero is clearly detected. This result agrees with the visual inspection of the Part A hard and soft band light curves (see Fig. 8). The flare detected in the hard band appears also in the soft band light curve, although with a smaller amplitude. The Part C CCF shows again a good correlation between soft and hard light curves, however in this case a significant


Fig. 17. Same as with the previous $C C F$ figures, for the orbit 171-2 light curves. In this case, in the middle panel we also plot the autocorrelation function of the "Part A" soft band light curve (dashed line).

positive lag is detected: the hard band variations are delayed with respect to the soft band variations, by $4.5 \pm 2 \mathrm{~min}$. The part B $C C F$ is very noisy. Overall, an anti-correlation "signal" can be observed. This is caused by the fact that the Part B light curves show different trends; the soft band flux increases while the hard band flux decreases (see Fig. 8).

In Sect. 3.4 we divided the orbit 171-2 light curves in two parts, A and B (Fig. 9). The Part A CCF (shown in the second panel from top in Fig. 17) implies that the soft and hard band light curves are well correlated $\left(C C F_{\max } \sim 0.9\right)$, and the soft band is delayed with respect to the hard band by $\sim 5.5 \pm 2 \mathrm{~min}$. In order to demonstrate the significance of the asymmetry in the $C C F$ (with respect to lag zero) which is caused by the presence of a negative lag, we show in the same panel the autocorrelation function $(A C F)$ of the soft band light curve (plotted as a dashed, black line). A comparison between the $C C F$ and $A C F_{\text {soft }}$ clearly demonstrates the asymmetry of the $C C F$ towards negative lags. At lags smaller than $\sim-300 \mathrm{~s}$, the soft band light curve is better correlated with the hard band light curve than with itself! The $C C F$ of Part B is shown in the lower panel of Fig. 17. Although it is noisy (for that reason we did not attempt a model fitting), a positive peak near zero lag is clearly evident. This is a rather surprising result, since the large amplitude, hard band flare of Part B appears to be absent in the soft band light curve. However the appearance of a peak in the $C C F$ indicates that the small amplitude variations in the soft band are associated with the hard band flare. In other words, the flare is present in the soft band as well, albeit with a much smaller amplitude. We believe that the hard/soft band positive correlation in this case was detectable through the high statistical significance of the XMM data. Only the large count rate in the soft band enables us to recognize the small amplitude variations associated with the hard band flare.

In Fig. 18 we plot the $C C F$ of the two parts (A and B) of the light curves of orbit 259 from Fig. 10. The $C C F$ of the total 


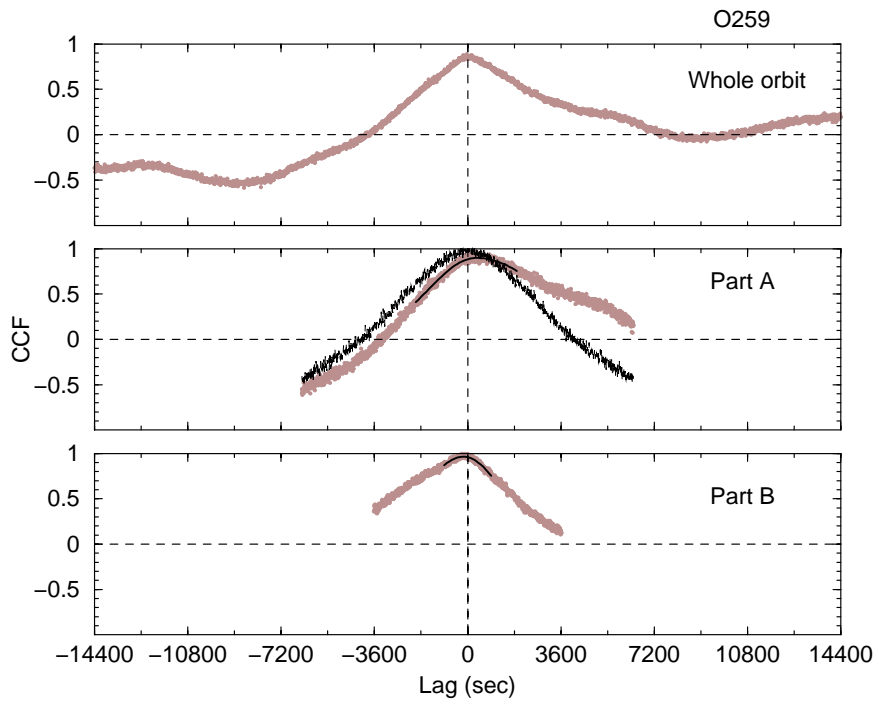

Fig. 18. Same as with Fig. 17 for the orbit 259 light curves. The dashed line in the middle panel shows the $A C F$ of the "Part A" soft band light curve.

light curve shows a large peak near zero lag, and an asymmetry towards positive lags. The part A and B light curves are also well correlated $\left(C C F_{\max } \sim 0.9\right)$, however significant delays are observed between the soft and hard band Part A light curves. We detect a positive lag of $\sim 5.5 \pm 2 \mathrm{~min}$, i.e. in this case the soft band variations lead the variations in the hard band light curve. This result is consistent with the light curves shown in Fig. 10. The soft band light curve appears to reach the "plateau" level of the flare faster than the hard band light curve. Towards the end of the observation, when the flare decays, the hard band light curve appears to enter this phase first. We do find a negative $k_{\max }$ in the Part B $C C F$, however, the significance of this result is less than $95 \%$.

As with the $C C F$ plot for orbit 171-2, in the middle panel of Fig. 18, together with the $C C F$, we also plot the $A C F_{\text {soft }}$ (dashed black line) of Part A. The comparison of the two functions clearly reveals the asymmetry of the $C C F$ towards positive lags. At positive lags (e.g. $k>1000$ s), the correlation of the soft band with the hard band light curve is stronger than the correlation with itself. It is interesting to compare the middle panel plots in Fig. 18 and Fig. 17. We believe that the comparison of the $C C F \mathrm{~s}$ with the $A C F_{\text {soft }}$ in these plots clearly demonstrates the differences between the two $C C F \mathrm{~s}$. The Part A, Orb 171-2 CCF is asymmetric towards negative lags $\left(k_{\max } \sim-5 \mathrm{~min}\right)$, while the opposite effect is observed in the Part A, orbit $259 C C F\left(k_{\max } \sim+5 \mathrm{~min}\right)$. In fact, when we performed the $C C F$ analyses of synthetic light curves for the computation of the uncertainties associated with $k_{\max }$ we did not obtain, in any run, a value of $k_{\max } \sim+5 \mathrm{~min}$ or $k_{\max } \sim-5 \mathrm{~min}$ in the cases of the CCFs of Part A, orbit 171-2 and 259 , respectively. We conclude that the differences between the $C C F$ s shown in the middle panels of Figs. 17 and 18 are statistically significant and imply that different physical parameters determine the observed variations in these cases.

Finally, the $C C F$ s of the whole length and Part A, B light curves of orbit 440-1 from Fig. 11 are plotted in Fig. 19. The
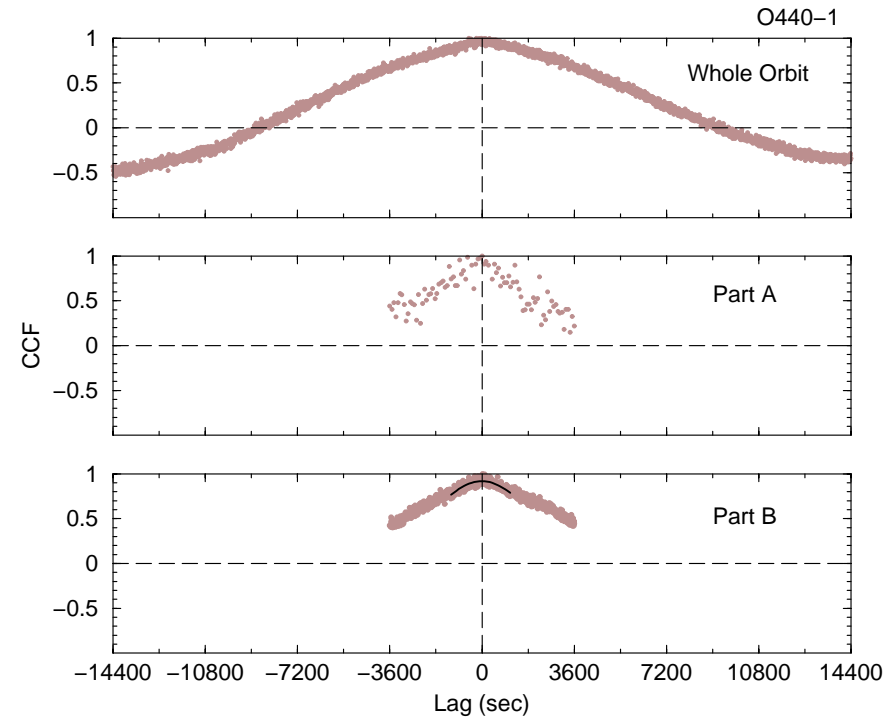

Fig. 19. The $C C F$ plots for the whole length hard and soft band light curves of orbit 440-1 (upper panel), and for Parts A and B of the light curves (middle and lower panel, respectively) shown in Fig. 11.

Part A $C C F$ is noisy. In fact, the use of the 8-s binned light curves for the calculation of the $C C F$ resulted in a very noisy plot. For that reason, the Part A $C C F$ shown in the middle part of Fig. 19 was computed using the 80-s binned light curves. Most probably, the reason for the large scatter is the presence of low amplitude variations in this part of the observation, combined with the fact that the source was at a very low flux state, hence the Poisson noise effects are pronounced ("destroying" any Cross-Correlation signals at different lags). Despite this fact (which prevents us from a proper model fitting), we can easily detect a positive peak near zero lag. The Part B $C C F$ is quite similar to the Part A $C C F$. It shows a good correlation between the two light curves and appears to be symmetric around zero lag. We do not find significant delays between the variations in the two light curves.

\section{Power spectrum analysis}

The large mean count rate of the XMM-Newton observations presented in this work, gives us the opportunity to investigate accurately the form of the X-ray power spectral density function (PSDF) of Mrk 421 at high frequencies (i.e. $v>10^{-5} \mathrm{~Hz}$ ). We computed the PSDF in both the soft and hard energy bands using RGS R1-R2 and PN 2-10 keV light curves, respectively.

For RGS we have selected the first order spectra (from 0.33 to $2.44 \mathrm{keV}$ ) and have generated light curves from them. Contrary to the PN the readout time of the RGS detector is relatively long (4.60 seconds when 8 CCDs are read out). To avoid numerical effects we have used a bin size for the light curves corresponding to the readout time. This takes also into account the spectral variation of the source over the CCD (each data point samples the full spectral shape). Only telemetry gaps cannot be accounted for and these data points had to be omitted.

The choice of the RGS light curves was motivated by the fact that, contrary to the soft band PN light curves, some of them (the RGS R1 light curves of the orbits 171-1 and 171-2, 


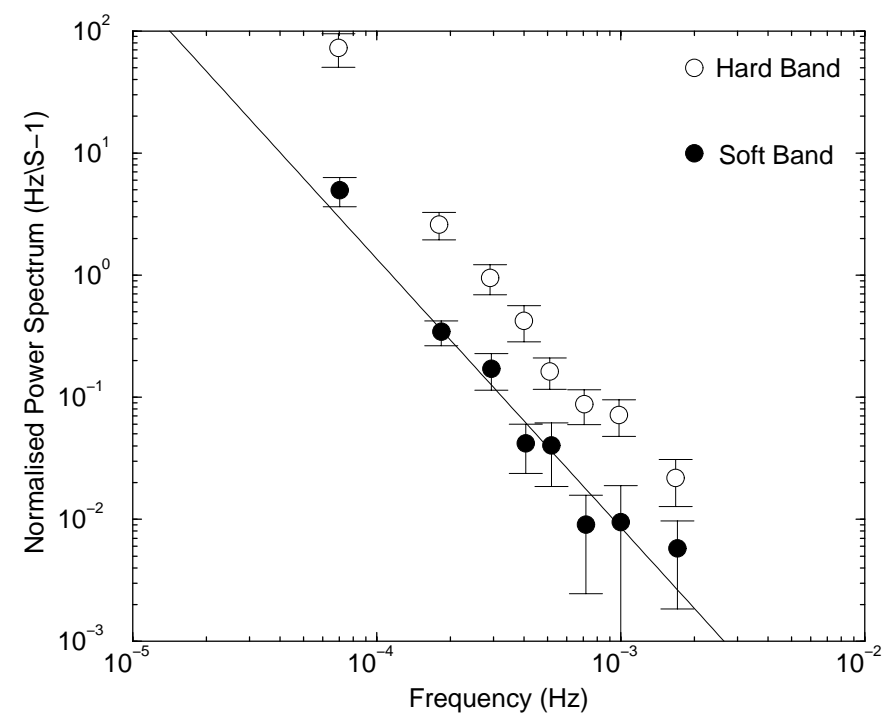

Fig. 20. Plot of the hard and soft band PSDF of Mrk 421 (empty and filled circles, respectively). The solid line shows the average ASCA/RXTE power spectrum of the source (from Kataoka et al. 2001).

and the RGS R2 light curves of the orbits 84, 171-1 and 171-2) have no missing points in them, even when we use a bin size as small as $4.6 \mathrm{~s}$ (the use of small bin size in the light curves results in better frequency resolution in the computed PSDF). For the estimation of the hard band PSDF we used the PN 80$\mathrm{s}$ binned light curves apart from the light curve of orbit 165 (there are two large gaps of missing points in it; see Fig. 7), orbit 440-1 (due to the large experimental noise the resulting PSDF is of low quality) and orbit 440-2 (which does not show any significant intensity variations). Because of the use of a large bin size, the resulting light curves are evenly sampled with no missing points in them.

For each light curve we computed its periodogram and normalized it to the square of the mean count rate (i.e. Papadakis \& Lawrence 1995). Typically, we could compute the PSDF at frequencies between $\sim 2 \times 10^{-5} \mathrm{~Hz}$ and $\sim 0.1 \mathrm{~Hz}$ (for the RGS data) and $\sim 6.5 \times 10^{-3} \mathrm{~Hz}$ for the PN data. However, despite the large count rate in the light curves, the Poisson noise component was dominating the resulting PSDF. In order to increase the signal to noise of the computed PSDFs, we combined all the RGS periodograms in one file and the $\mathrm{PN}$ periodograms in a separate file. We sorted the files in order of increasing frequency, subtracted the expected level of the Poisson statistics power, and then grouped them into bins of size 200. The resulting soft and hard band PSDFs of Mrk 421 are shown in Fig. 20 (filled and open squares, respectively). Using standard $\chi^{2}$ statistics we fitted a power law model of the form $P_{v}=A v^{-\alpha}$, separately to the hard and soft energy band PSDF. The best fitting slope values are $\alpha_{\mathrm{HB}}=2.6 \pm 0.15$ and $\alpha_{S F}=2.5 \pm 0.17$ (where with $\alpha_{\mathrm{HB}}$ and $\alpha_{S B}$ we denote the slope of the hard and soft energy band PSDFs, respectively). In Fig. 20 we also plot the average $A S C A$ PSDF of the source (solid line) as computed by Kataoka et al. (2001). The agreement between the ASCA and the XMM-Newton PSDFs is very good in the sense that their slopes are almost identical. The hard band PSDF has a larger normalization when compared to the soft band PSDF. This is expected since the amplitude of the variations is larger in the hard band light curves. The ASCA PSDF was based on broad band energy (i.e. $0.5-10 \mathrm{keV}$ ) light curves. Comparison of the average XMM-Newton PSDF with the ASCA PSDF shows that the former has a slightly larger normalization. Perhaps then the amplitude of the X-ray PSDF of Mrk 421 varies but its shape remains constant.

\section{Discussion}

In this work we present a spectral and timing analysis of eight calibration phase XMM-Newton observations of Mrk 421. The superior quality of the present light curves allows a detailed investigation of the the flux and spectral variability properties of the source on short time scales (i.e. between $\sim 10^{2}-5 \times 10^{4} \mathrm{~s}$ ). The source shows significant, medium amplitude (i.e. $\sim 5 \%-$ $20 \%$, on average) flux variations in all cases, except during the orbit 440-2 observation, when it was at its lowest flux state. Both, long term trends with time scales comparable to or even longer than the observation lengths and flare like events, which last $\sim$ few ksec, are observed. The amplitude of the hard band $(2.4-10 \mathrm{keV})$ variations is systematically larger than the soft band (0.2-0.8 keV) amplitude.

Due to the currently still existing calibration uncertainties of the PN detector response at low energies, we are not able to reliably fit complex models to the source spectrum in the total energy band. However, despite the uncertainties, it is clear that two models which are currently used to describe the X-ray spectra of BL Lac objects, the "broken power law" and the "continuously curved" models, can not characterize adequately the observed spectra. At least one more component is necessary in order to fit successfully the full $0.2-12 \mathrm{keV}$ band energy spectrum of Mrk 421. When the current uncertainties in the detector response are resolved, the unique quality of the XMM-Newton data will allow to study the X-ray spectra of BL Lacs in a way that was not possible so far with the data from previous satellites.

In order to study the complex spectral variations of the source we used hardness ratio plots. We also computed $C C F \mathrm{~s}$ in order to investigate the temporal cross-links between the soft and hard band light curves, using both the total light curves, and various sub-parts, chosen according to the results from the hardness ratio study. The main conclusions from the hardness ratio and $C C F$ analysis of the present XMM-Newton observations of Mrk 421 are as follows:

1) The flux variations are associated with significant spectral variations as well. In all cases, apart from Part $\mathrm{C}$ in orbit 171-1 (see below), the spectrum hardens/flattens when the source flux increases, and vice versa.

Currently, we are not able to identify reliably the individual spectral components which are responsible for the observed spectral variations. Nevertheless, using the results from a broken power law model fit to the $1.5-12 \mathrm{keV}$ spectra of various sub-parts of the orbit 165 and 171-1 observations (these observations show the smallest and largest amplitude $H R$ variations, respectively) we find that the spectral variations are mainly caused by two facts: (a) the normalization of the hard power 


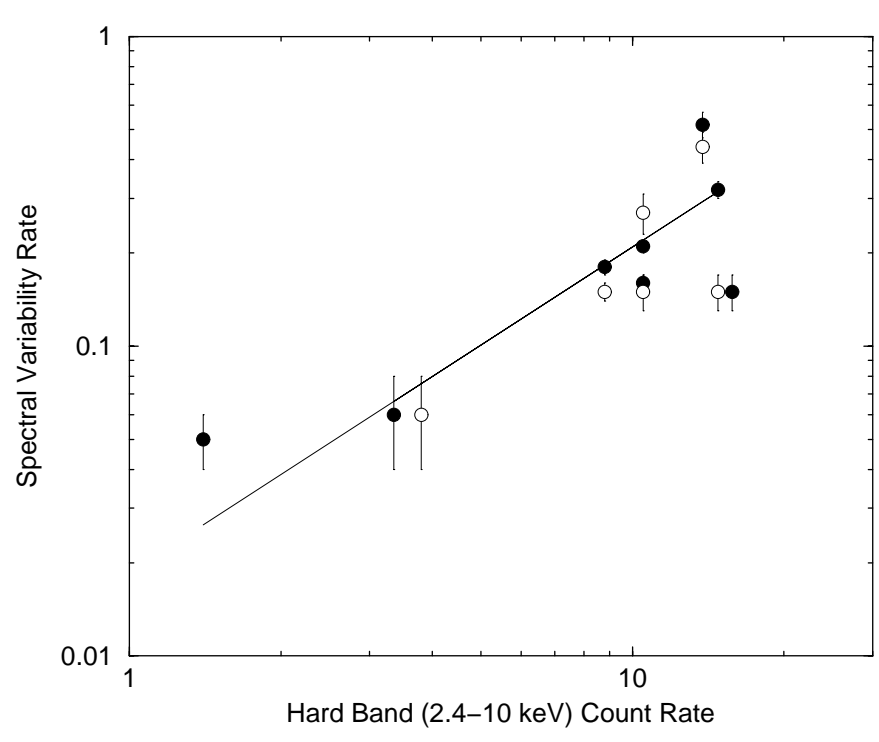

Fig. 21. Rate of spectral variability as a function of the count rate in the 2.4-10 keV energy band; open circles indicate time periods where the spectrum softens, filled circles when it hardens.

law shows larger amplitude variations, (b) the spectral slopes of both the low and high energy band decrease (i.e. the spectrum flattens) as the source flux increases. Surprisingly, we find that the break energy remains relatively constant during the flux variations. However, these results can only be considered as suggestive, until a correct description of the spectral shape over the total energy range has been obtained. Then the XMM-Newton data might not only allow us a different view to the overall energy spectrum of the source (see above), but also to the component(s) that are responsible for the spectral variations.

2) The spectral variability rate is not the same in all cases. In other words, the spectrum hardens/softens at a rate which varies from observation to observation - perhaps as a function of the intensity of the source. In Fig. 21 we have plotted the $\mathcal{V}_{\mathrm{s}}$ as function of the hard band count rate, except for the orbit 84 - timing mode observation. The choice of the hard band count rate was motivated by the fact that we are then less affected by the use of the thin filter in the orbit 259 observation. Filled circles correspond to $\mathcal{V}_{\mathrm{s}}$ values when the source flux decreases, open circles to the rising parts of the observations (for orbits 165 and 171-1 the two values are identical, we therefore slightly offset the points in the plot for clarity). It appears that the SVR values are positively correlated with the source intensity: as the source flux increases, the spectral variability rate increases as well. Application of Kendall's $\tau$ test shows that the probability of a false correlation (i.e. correlation by chance) is less than $1 \%$. In fact, the correlation may be even stronger, considering the fact that there is some uncertainty in the hard band count rate (see discussion in Sect. 2.1). A power law with a slope of $\sim 1$, shown as solid line in the figure, fits the data quite well.

3) The cross-correlation analysis of the total light curves shows that, in some cases, the hard and soft band variations are well correlated near zero lag (i.e. orbit 84(tim), orbit 84, orbit 259, and orbit 440-1), but not in all (orbit 165: broad peak at negative lags, orbit 171-1: anti-correlation at all lags up to \pm 4 hrs, orbit 171-2: $C C F$ peak near zero lag, but reduced in amplitude).

3a) The results are modified significantly when subintervals, based on the absence/presence of strong flares and/or well defined flux variability "trends" (like flux rise/decay) are used instead of the total light curves. We find that in some cases the soft and hard band light curves are well correlated, with no measurable delay: Part B in orbit 84 (tim) and orbit 84, Part A in orbit 165 and orbit 171-1, and Part B of orbit 259 and orbit 440-1. All these parts are associated with a single flare-like event (or double, as in the case of Part A, orbit 171-1) with a duration less than $\sim 15 \mathrm{ksec}$. The $C C F$ s have a "triangular", symmetric shape, with sometimes a moderate $\left(C C F_{\max } \sim 0.5-0.7\right)$, in other cases a strong $\left(C C F_{\max } \sim 0.95\right)$ peak.

$3 \mathrm{~b})$ In other cases, the hard band variations are leading the soft band variations: Part A in orbit 84(tim) and orbit 84, Part B of orbit 165 and Part A in orbit 171-2. The $C C F$ s show a broad, strong peak $\left(C C F_{\max } \sim 0.9\right)$ and the delay is of the order of $\sim 5 \mathrm{~min}$. In all cases, the probability that $k_{\max }<0$ is higher than $95 \%$.

$3 c$ ) In two cases (namely Part C, orbit 171-1, and Part A, orbit 259), we observe an opposite behavior: the soft band leads the hard band variations. The $C C F$ s look similar to those we observe the negative lags, with similarly strong peaks, but located at $k_{\max } \sim+5 \mathrm{~min}$.

The parts of the light curves which do show significant lags have typical durations of more than $\sim 15 \mathrm{ksec}$ and they show long term trends like a flux increase or decrease. The fact that those parts with no significant delays have durations less than $\sim 15 \mathrm{ksec}$ suggests that the lack of detectable delays is associated with the length of the light curve parts: there might be lags of similar magnitude, but the light curves are not long enough to detect them (in some cases the $C C F$ peak is indeed low). However, we believe that this is not the case for the following reasons: (a) the shape of the $C C F$ of the parts with no significant delays is different from the $C C F$ of those which do show delays. Of course, different realizations of a red noise process (like the Mrk $421 \mathrm{X}$-ray variability) can result in differently shaped $C C F \mathrm{~s}$ by chance. However, the fact that all $C C F$ s with measurable lag show an almost identical shape, which is very different from the shape of the $C C F \mathrm{~s}$ where we do measure a delay, is rather suggestive for the assumption that their difference is intrinsic. (b) The $k_{\max }$ values of the parts with no detectable lags are smaller than the respective values of the parts which show delays. If the non-detection of lags was caused by the small length of the light curves, we would expect the $k_{\max }$ values to be similar in all cases, with larger errors for the parts with small duration. This is not the case, which indicates that the difference in the length of the light curves does not seriously affect the detection of lags. We therefore believe that the difference is intrinsic: either shorter flares do not show any delays, or the delays are coupled to the flare's duration, i.e. the shorter the flare, the smaller the delay.

3d) Finally, in three cases, namely Part C of orbit 165 , Part B of orb 171-1, and orbit 440-2, the source behaves in an unusual way. In the first two cases, the computed $C C F$ s are noisy and show no well defined peaks. The light curves appear 
to be un-correlated during these periods, which last $\sim 7 \mathrm{ksec}$ and $\sim 15 \mathrm{ksec}$, respectively. The variations are of low amplitude, and while the soft band flux increases, the hard band flux either remains constant or even decreases. In the case of orbit 440-2, the source is in a very low state and no significant variations can be detected at all.

4) The PSDF of Mrk 421 shows a "red noise" character in the frequency range $7 \times 10^{-5}-2 \times 10^{-3} \mathrm{~Hz}$. No periodicities or other characteristic time scales could be detected, and a power law model with a slope of -2.5 could fit well both the soft and hard energy band PSDFs. This result is consistent with the results from previous work based on data from EXOSAT, GINGA, and ASCA (Kataoka et al. 2001, and references therein).

\subsection{Comparison with previous work}

As discussed in the introduction, Mrk 421 has been extensively observed by all previous X-ray missions. Our results are in agreement with the results of Brinkmann et al. (2001) and Sembay et al. (2002), which were based on the analysis of a subset of the XMM-Newton observations presented in this work. We do not detect significant delays when we consider the total light curves, and the spectral variations we find are similar to what was observed by Sembay et al. (2002). For example, they see that the source becomes "softer" as the flux decreases during the orbit 171 observation, in agreement with our results from both our $H R$ plot and spectral model fitting analyses. On the other hand, they find no spectral variability during the orbit 84 observation, in contrast to the significant $H R$ plot variations we observe (see Fig. 6). This is probably due to the fact that their analysis is based on the results of single power law fits to the $2-10 \mathrm{keV}$ energy spectrum which is, as we show in this work, a rather poor approximation of the true shape of the spectral energy distribution at these energies.

Previous observations of Mrk 421 have claimed significant "hard" or "soft" lags (where the hard band lag or lead the soft band variations, respectively), e.g. Takahashi et al. (2000), Fossati et al. (2000). In a way, our results confirm these claims. However, the lag values we find $(\sim \pm 5 \mathrm{~min})$ are much smaller than the values reported in the past $(\sim 0.5-1.5$ hours). If these delays are "real" and not the result of systematic biases due to the uneven sampling pattern of the light curves, the difference in the lag values may be due to the fact that the delays determined in the past were based on observations which showed flares with time scales longer than the flares detected in the present XMM-Newton observations. Perhaps then, as we have already mentioned, the lag size depends on the time scale of the "event" associated with it.

\subsection{Implications for physical time scales}

The detection of delays between the soft and hard band variations can give us information on whether the acceleration or the cooling time scale controls the observed variations. The detection of "soft" lags is usually interpreted as an indication that the cooling process dominates the system. If the acceleration

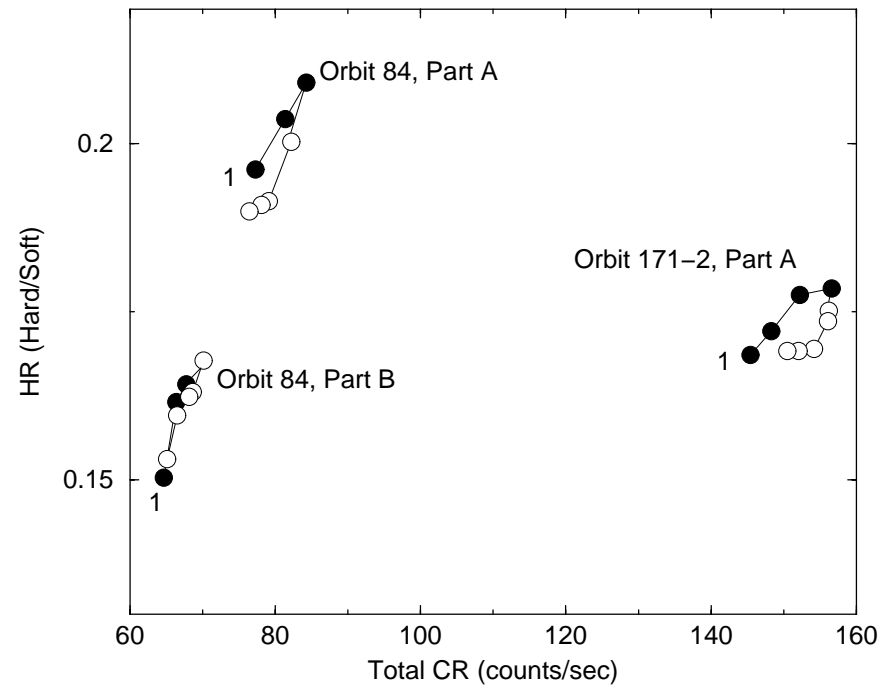

Fig. 22. Spectral evolution of three particular flares as function of the total count rate. The flares start at the labels " 1 "; filled circles indicate the rising times of the flares, open circles the decaying times.

time scale is much smaller than the cooling time scale, information on the acceleration is suppressed by cooling, emission propagates from higher to lower energy and the higher energy lead the lower energy photons. If the acceleration is comparable to the cooling time scale, the acceleration process dominates, emission propagates from lower to higher energy and the lower energy lead the higher energy photons (the case of "hard lags"). The fact that in some cases we observe soft and in other cases hard delays implies that the difference between the acceleration and/or cooling time scales of the particles emitting in the different energy bands is not always the same. One possibility is that the acceleration process does not operate in a unique way. In the case of shocks, for example, the shock formation and the subsequent particle acceleration may not be similar at all times, or we observe emission from multiple "shocked" regions which have different physical parameters.

If there are delays between the variations observed in different energy bands, we should be able to observe characteristic "loop"-like structures in the $H R$ plots. We find soft delays in four cases, of which two correspond to a simple flux increase (Part A of orbit 84, timing mode, and Part B of orbit 165). Qualitatively, these light curve parts could correspond to a situation where the acceleration is almost instantaneous and the cooling time scale is comparable to the light crossing time of the source. In this case, the flux increases as the observer receives radiation from an increasing volume. The hard band electrons, after some cooling time, start emitting in the soft band as well, and the delay of the soft band photons is proportional to the difference in the cooling time scale of the particles that emit at the hard and soft energy bands. In the other two parts (Part A of orbit 84, and Part A of orbit 171-2) we observe two well sampled flares (at the beginning of orbit 84, and after $\sim 10 \mathrm{ksec}$ from the start of orbit 171-2 observations), lasting for $\sim 10 \mathrm{ksec}$ each. In Fig. 22, we plot the $H R$ values for the duration of the flares. We have used the data shown in Figs. 6 and 9 which correspond to the two flares, and binned 
them using a bin size of 15 , in order to increase the signal to noise. Filled/open circles show the spectral evolution during the rise/decay parts of the flare, respectively (the starting point is indicated with the number " 1 "). The $H R$ variations, although statistically significant, are very small in amplitude. In both cases, a roughly quasi-circular pattern appears in the $H R$ plot, and the spectral evolution follows the loop in a clockwise direction. This is consistent with the fact that the hard band lead the soft band photons during the flare evolution.

In the first case where we detect a hard lag, we observe the total flux decaying and then rising again (Part $C$ in orbit 171-1) and in the second case we observe a flare like event with an extended "plateau" state (orbit 259). In the former case, the spectral variations follow an anti-clockwise loop in the $H R$ plot, as shown clearly from the points plotted the lower panel in Fig. 8. As the source flux decreases the spectrum steepens (filled circles in the lower left part of the $H R$ plot) and then flattens as the flux increases once more (crosses in the same part of the plot). However, the $H R$ points during the rise part lie systematically below the points of the decaying part, indicating a spectral evolution in the anti-clockwise direction. In the latter case, the $H R$ variations during the flux rising and decaying part of the flare (at the beginning and the end of the observation; crosses and filled circles in Fig. 10) clearly show a loop like pattern. As the flux evolves from rise to decay the $H R$ values move in the anti-clockwise direction. This is exactly what is expected in the case of hard lags. We conclude that the detection of hard or soft lags is consistent with the observed spectral variations in the respective parts of the observations.

What about the parts where we do not detect any significant delays? For example, well sampled flares like those in orbit 165, Part A and orbit 84, Part B, do not show any significant delays between the variations in the two energy bands. In Fig. 22, we also plot the binned $H R$ variations which correspond to the flare seen in orbit 84 , Part B. In this case, the spectral evolution during the rising and decaying part of the flare follows an almost identical path in the $H R$ plot. Perhaps both, the acceleration and cooling time scales, are very small and thus differences between the time scales of the electrons which emit in the hard and soft band cannot be detected as time lags, and the flare evolution is in this case determined by the light crossing time scale.

\section{Conclusions}

We have presented an analysis of the currently available XMM-Newton observations of Mrk 421, relying on the PN and RGS data. The temporal and spectral behavior of the source is very complex. In general, an increase in flux is accompanied by a hardening of the spectrum as expected from shift of the Synchrotron peak to higher energies. But there are exceptions and the rate of the spectral changes varies strongly. The shortest variability time scales appear to be of the order of $\gtrsim \mathrm{ksec}$; the longest time scales could not be resolved in any of the observations. The lags between the hard and soft band flux are small and can be of different sign. Correspondingly, it appears hard to deduce uniquely the underlying physical parameters for the emission process from the observations. For the currently favored "shock-in-jet" model for the BL Lac emission (see, for example, Spada et al. 2001) this implies that we are seeing the emission from multiple shocks which have either largely different physical parameters or that we detect the emission from similar shocks at very different states of their evolution, heavily confused by relativistic beaming and time dilatation effects. More observations with longer exposures, more sophisticated (perhaps non-linear) data analysis methods and explicit jet simulations are required for a better understanding of these objects.

Acknowledgements. This work is based on observations with XMMNewton, an ESA science mission with instruments and contributions directly funded by ESA Member States and the USA (NASA).

\section{References}

Bregman, J., Maraschi, L., \& Urry, C. M. 1987, in Exploring the Universe with the IUE Satellite, ed. Y. Kondo (Dordrecht: Reidel), 685

Brinkmann, W., Sembay, S., Griffiths, R. G., et al. 2001, A\&A, 365, L162

Chiaberge, M., \& Ghisellini, G. 1999, MNRAS, 306, 551

Edelson, R., Griffiths, G., Markowitz, A., et al. 2001, ApJ, 554, 274

Fossati, G., Chiappetti, L, Celotti, A., et al. 1998, Nucl. Phys. B. Proc. Supp. 69/1-3,

Fossati, G., Celotti, A., Chiaberge, M., et al. 2000a, ApJ, 541, 153

Fossati, G., Celotti, A., Chiaberge, M., et al. 2000b, ApJ, 541, 166

Kataoka, J., Takahasi, T., Makino, F., et al. 2000, ApJ, 528, 243

Kataoka, J., Takahashi, T., Wagner, S. J., et al. 2001, ApJ, 560, 569

Macomb, D. J., Akerlof, C. W., Aller, D. H., et al. 1995, ApJ, 449, L99

Makino, F., Fink, H. H., \& Clavel, J. 1992, in Frontiers of X-ray Astronomy, ed. Y. Tanaka, \& K. Koyama (Tokyo: Univ. Acad. Press), 543

Malizia, A., Capalbi, M., Fiore, F., et al. 2000, MNRAS, 312, 123

Maraschi, L., Fossati, G., Tavecchio, F., et al. 1999, ApJ, 526, L81

Papadakis, I., \& Lawrence, A. 1995, MNRAS, 272, 161

Pian, E. 2002, Publ. Astr. Soc. Austr. 19, in press [astro-ph/0203423]

Punch, M., Akerlof, C. W., Cawley, M. F., et al. 1992, Nature, 358, 477

Sembay, S., Edelson, R., Markowitz, A., Griffiths, R. G., \& Turner, M. J. L. 2002, ApJ, 574, 634

Spada, M., Ghisellini, G., Lazzatti, D., \& Celotti, A. 2001, MNRAS, 325,1559

Takahashi, T., Tashiro, M., Madejski, G., et al. 1996, ApJ, 470, L89

Takahashi, T., Kataoka, J., Madejski, G., et al. 2000, ApJ, 542, L105

Tanihata, C. 2002, Ph.D. Thesis Tokyo Univ., ISAS Research Note, 739

Tanihata, C., Urry, C. M., Takahashi, T., et al. 2001, ApJ, 563, 569

Tashiro, M. 1994, Ph.D. thesis, Univ. of Tokyo; ISAS Research Note, 549

Tavecchio, F., Maraschi, L., \& Ghisellini, G. 1998, ApJ, 509, 608

Urry, C. M., \& Padovani, P. 1995, PASP, 107, 803

Zhang, Y. H., Celotti, A., Treves, A., et al. 1999, ApJ, 527, 719 